Publ. Mat. 66 (2021), 129-158

DOI: 10.5565 /PUBLMAT6612105

\title{
MONOTONE SYSTEMS INVOLVING VARIABLE-ORDER NONLOCAL OPERATORS
}

\author{
Miguel YANGARI
}

\begin{abstract}
In this paper, we study the existence and uniqueness of bounded viscosity solutions for parabolic Hamilton-Jacobi monotone systems in which the diffusion term is driven by variable-order nonlocal operators whose kernels depend on the space-time variable. We prove the existence of solutions via Perron's method, and considering Hamiltonians with linear and superlinear nonlinearities related to their gradient growth we state a comparison principle for bounded sub and supersolutions. Moreover, we present steady-state large time behavior with an exponential rate of convergence.
\end{abstract}

2010 Mathematics Subject Classification: 49L25, 35K41, 60J75, 47G20.

Key words: viscosity solutions, Hamilton-Jacobi, variable-order nonlocal operators, comparison principles, large time behavior.

\section{Introduction}

In this paper we are interested in studying the existence and uniqueness of systems with the form

$$
\partial_{t} u_{i}+H_{i}\left(x, t, \mathbf{u}(x, t), D u_{i}(x, t), D^{2} u_{i}(x, t), \mathcal{T}_{i, x, t} u_{i}(x, t)\right)=0 \quad \text { in } Q,
$$

$i \in I(n)$, where $Q:=\mathbb{R}^{d} \times(0,+\infty)$ and $I(n):=\{1,2, \ldots, n\}$. We have considered here the usual notation of bold letters for vectors.

We complement system (1.1) with the initial condition

$$
\mathbf{u}=\mathbf{u}_{0}:=\left(u_{0, i}\right)_{i \in I(n)}
$$

of continuous functions, that is, $u_{0, i} \in C\left(\mathbb{R}^{d}\right)$ for all $i \in I(n)$.

Moreover, for each $i \in I(n), H_{i} \in C\left(\mathbb{R}^{d} \times \mathbb{R}_{+} \times \mathbb{R}^{n} \times \mathbb{R}^{d} \times \mathbb{S}^{d} \times \mathbb{R}\right)$, and the term $\mathcal{T}_{i, x, t}$ (which for ease of notation we call only $\mathcal{T}_{i}$ ) is a nonlocal operator playing the role of the diffusion defined as follows: for $x \in \mathbb{R}^{d}$, $t \in \mathbb{R}_{+}$, and $\phi: \mathbb{R}^{d} \rightarrow \mathbb{R}$, a bounded continuous function which is $C^{2}$ in a neighborhood of $x$, we write

$$
\mathcal{T}_{i} \phi(x, t)=\int_{\mathbb{R}^{d}}\left[\phi(x+z)-\phi(x)-\mathbb{1}_{B}(z)\langle D \phi(x), z\rangle\right] K_{i}(x, t, z) d z,
$$


where $B$ is the open ball centered at zero with radius 1 . For each $i \in I(n)$, the kernel $K_{i}: \mathbb{R}^{d} \times \mathbb{R}_{+} \times\left(\mathbb{R}^{d} \backslash\{0\}\right) \rightarrow \mathbb{R}$ is a continuous function, satisfying

$$
0 \leq K_{i}(x, t, z) \leq \frac{C_{i}}{|z|^{d+2 \alpha_{i}(x, t, z)}},
$$

and $\alpha_{i}: \mathbb{R}^{d} \times \mathbb{R}_{+} \times \mathbb{R}^{d} \rightarrow(0,1)$ is a continuous function such that

$$
\begin{aligned}
0<\alpha^{-} & :=\min _{(x, t, z, i) \in \mathbb{R}^{d} \times \mathbb{R}_{+} \times \mathbb{R}^{d} \times I(n)} \alpha_{i}(x, t, z) \\
& \leq \max _{(x, t, z) \in \mathbb{R}^{d} \times \mathbb{R}_{+} \times \mathbb{R}^{d} \times I(n)} \alpha_{i}(x, t, z)=: \alpha^{+}<1 .
\end{aligned}
$$

Since for each $i \in I(n)$ the operators $K_{i}(\cdot)$ and $\alpha_{i}(\cdot)$ are functions depending on the $(x, t, z)$-variable, following the ideas of $[\mathbf{1 2}],[\mathbf{4 6}]$, the terms $\mathcal{T}_{i}$ are called variable-order nonlocal operators. It is interesting to note that under the symmetric assumption on $K_{i}$ we can remove the compensator term of (1.3). Hence, considering $K_{i}(x, t, z)=C_{i}|z|^{-\left(d+2 \alpha_{i}(x, t, z)\right)}$ with $\alpha_{i}$ verifying $\alpha_{i}(x, t, z)=\alpha_{i}(x, t,-z)$ for all $(x, t, z) \in \mathbb{R}^{d} \times \mathbb{R}_{+} \times \mathbb{R}^{d}$, a particular class of operators with the form (1.3) are the variable-order fractional Laplacians given by the expression

$$
\mathcal{T}_{i} u(x, t)=\int_{\mathbb{R}^{d}}[\phi(x+z)-\phi(x)] \frac{C_{i}}{|z|^{d+2 \alpha_{i}(x, t, z)}} d z .
$$

Moreover, when $\alpha_{i} \in(0,1)$ is a constant, $\mathcal{T}_{i}$ reduce to the usual fractional Laplacian operator.

As general conditions we assume that the Hamiltonians satisfy the following properties:

(H1) Degenerate ellipticity: for all $i \in I(n)$ and $(x, t, \mathbf{r}, p) \in \mathbb{R}^{d} \times \mathbb{R}_{+} \times$ $\mathbb{R}^{n} \times \mathbb{R}^{d}$

$$
H_{i}\left(x, t, \mathbf{r}, p, X, l_{1}\right) \leq H_{i}\left(x, t, \mathbf{r}, p, Y, l_{2}\right) \quad \text { if } l_{2} \leq l_{1}, Y \leq X .
$$

(H2) Monotone property: there exists $c_{i j} \in \mathbb{R}$ for all $i, j \in I(n)$ such that $\sum_{j=1}^{n} c_{i j} \geq c_{0}$ with $c_{0} \geq 0$ for all $i \in I(n)$ and for any $(x, t, \mathbf{r}, p, X, l) \in$ $\mathbb{R}^{d} \times \mathbb{R}_{+} \times \mathbb{R}^{n} \times \mathbb{R}^{d} \times \mathbb{S}^{d} \times \mathbb{R}$ and $\delta>0$, we have

$c_{i j} \delta \leq H_{i}\left(x, t, \mathbf{r}+\delta \mathbf{e}_{j}, p, X, l\right)-H_{i}(x, t, \mathbf{r}, p, X, l) \leq 0, \quad$ if $j \neq i$, $c_{i i} \delta \leq H_{i}\left(x, t, \mathbf{r}+\delta \mathbf{e}_{i}, p, X, l\right)-H_{i}(x, t, \mathbf{r}, p, X, l)$, where $\left(\mathbf{e}_{i}\right)_{i \in I(n)}$ is the canonical basis of $\mathbb{R}^{d}$. 
Note that in (H2) necessarily $c_{i j} \leq 0$ for $i \neq j$ and $c_{i i} \geq 0$. A particular case of monotone systems verifying (H2) are the weakly coupled systems with the form

$$
H_{i}\left(x, t, \mathbf{u}, D u_{i}, D^{2} u_{i}, \mathcal{T}_{i} u_{i}\right):=h_{i}\left(x, t, u_{i}, D u_{i}, D^{2} u_{i}, \mathcal{T}_{i} u_{i}\right)+\sum_{j=1}^{n} c_{i j}(x, t) u_{j},
$$

for all $i \in I(n)$, where

$$
c_{i i}(x, t) \geq 0, c_{i j}(x, t) \leq 0 \text { for } j \neq i \text { and } \sum_{j=1}^{n} c_{i j}(x, t) \geq 0 .
$$

Concerning the classical local problems of first or second order, the basic assumptions (H1) and (H2) in the nonlocal-term independent formulation make it possible to deal with system (1.1)-(1.2) in the viscosity solution framework; see [23] and [25]. It is well known that this type of systems is compatible with comparison principles, those that together with some regularity properties lead to results related to homogenization, ergodicity, and large time behavior of first and second-order monotone and weakly coupled systems of Hamilton-Jacobi equations; see [18], [34], $[\mathbf{3 8}],[40]$, and references therein.

The notion of viscosity solutions was first introduced by Crandall and Lions [22] and their theory was applied initially to local partial differential equations, but motivated by applications to finance, physical sciences, and mechanics (see for example [15] and [52]), the theory was almost immediately extended to the context of partial integrodifferential equations, i.e., equations involving nonlocal operators such as Lévy operators and their best-known representative, the fractional Laplacian. There is a close connection with probability since nonlocal operators of Lévy type arise as the infinitesimal generator of stochastic Lévy processes and also appear in the context of optimal control of jump diffusion processes; see [16], [42], and [44].

The first paper devoted to the mentioned extension, in the context of stochastic control of jump diffusion processes, was developed by Soner $[\mathbf{4 7}]$. Following this work, Sayah [45], in the stationary case and using first-order equation techniques, studied stability, comparison results, and the existence of viscosity solutions of a quite general class of integrodifferential equations, nonlinear with respect to the nonlocal term. In the case of bounded measures, Alvarez and Tourin ([1]) obtained quite general results for parabolic equations. For singular measures several results were obtained, for instance in [5] and [43]. Jakobsen and Karlsen ([29], [30]) developed a general theory for second-order parabolic nonlinear integro-differential equations, including comparison results, continuous dependence estimates, and a maximum principle. For more advanced 
theory, concerning regularity, homogenization, and large time behavior of Hamilton-Jacobi problems, we refer to [2], [7], and [48]. It should be noted that the difficulties in the study of the above problems involving Lévy-type operators are, for example, the coupling of second-order derivatives and nonlocal terms, the singularity of the measure appearing in the nonlocal operator, and the lack of basic differentiation tools, such as the product or chain rule. These difficulties were extensively analyzed by Barles and Imbert in $[\mathbf{6}]$.

Concerning nonlocal operators of variable order which verify similar conditions to (K)-(S), several properties have been intensively investigated both from the probabilistic and the analytic point of view. Stablelike processes were introduced originally by Bass $[\mathbf{9}],[\mathbf{1 0}]$, by showing the uniqueness of solutions to the martingale problem as variants of $\alpha$-stable processes (said papers considered a spatially dependent index $\alpha(x)$ ). Such a process may be thought of as one that at the point $x$ behaves like the symmetric stable process of index $\alpha(x)$, but the index $\alpha(x)$ varies from point to point. Tsuchiya $([\mathbf{4 9}])$ defined the processes in terms of stochastic differential equations with jumps. In [28] Jacob and Leopold constructed a Feller semigroup generated by operators with variableorder symbols. Their approach was mainly based on the method of Jacob concerning the generation of Feller semigroups by pseudo-differential operators in $[\mathbf{2 6}],[\mathbf{2 7}]$, and the results of Leopold on pseudo-differential operators of variable order and corresponding function spaces $[\mathbf{3 5}],[\mathbf{3 6}]$, and $[\mathbf{3 7}]$. See also $[\mathbf{3 1}]$ and $[\mathbf{4 1}]$ for further results concerning the existence of transition densities and path behavior of stable-like processes.

On the other hand, there are numerous results related to problems in PDE's that involve nonlocal operators like (1.3). To mention just one example, using probabilistic methods, in [14] Bass and Levin proved Harnack inequality of harmonic functions with respect to a class of pure jump Markov processes in $\mathbb{R}^{d}$, whose kernels are comparable to those of symmetric stable processes. Bass and Kassmann generalized this result and obtained Hölder continuity of harmonic functions of variable order in $[\mathbf{1 2}]$ and $[\mathbf{1 3}]$. Bass also established in [11] the Schauder estimates for stable-like operators in $\mathbb{R}^{d}$. With a purely analytical proof, in [46], Silvestre provided regularity results for solutions to integrodifferential equations, including the case of an operator with variable orders. In [17], Caffarelli and Silvestre generalized this result to fully nonlinear integro-differential equations associated with symmetric kernels comparable to fractional Laplacian, and in [32] and [33], Kim and Lee extended this result to equations associated with nonsymmetric kernels. In [3], Bae proved regularity results for solutions of fully nonlinear 
integro-differential equations with variable-order operators. Also, in [4], Bae and Kassmann established Schauder estimates for problems involving variable-order operators. For other related works, we refer the reader to $[\mathbf{2 0}],[\mathbf{2 1}],[\mathbf{3 9}],[\mathbf{5 0}]$, and references therein. Especially, we mention these new works $[\mathbf{5 1}],[\mathbf{5 3}]$, where the order of the kernel in the variableorder fractional Laplacian verifies $(\mathrm{K})-(\mathrm{S})$ and depends continuously on the variables involved.

The aim of this work is to study the existence and uniqueness of bounded viscosity solutions for the parabolic Hamilton-Jacobi monotone system (1.1)-(1.2) in the framework of viscosity solutions, in which the integro-differential operators are given by (1.3). Moreover, we state a comparison principle for bounded sub and supersolutions and we give steady-state large time behavior with an exponential rate of convergence.

Let us briefly outline the content of this paper. Concerning system (1.1), the novelty is the presence of nonlinearities $H_{i}$ having an explicit interaction with the time variable. Also, the diffusion terms are driven by variable-order nonlocal operators whose kernel depends continuously on the space-time variable. As is usual in the viscosity theory, the existence of discontinuous solutions to (1.1) is performed by Perron's method, adapted in this case to systems involving nonlocal operators with $(x, t)$-dependent kernels satisfying $(\mathrm{K})-(\mathrm{S})$.

Since we are working with monotone systems and all the kernels $K_{i}$ verify the integrability condition (K1), which allows us to deal with the kernel dependency on the $(x, t)$-variables (see Section 4 ), following the standard procedure of doubling variables it is possible to provide a comparison principle for system (1.1). It is important to note that the continuity of the kernels $K_{i}$ and the orders $\alpha_{i}$ are crucial when proving the existence of solutions and the comparison principle. In addition, (K)-(S) allows the integro-differential operators $\mathcal{T}_{i}$ to verify the Lévy integrability condition (see [6]), allowing in certain cases the integrability of the kernel. Moreover, to prove the comparison result we concentrate on two main classes of Hamiltonians which are standard in the analysis of fully nonlinear equations, and have been addressed in different contexts to get well-posedness; see for example [6]. The first type of operators are Hamilton-Jacobi functionals with model form

$$
\begin{array}{r}
H_{i}(x, t, \mathbf{r}, p, X, l)=\sup _{\theta \in \Theta}\left\{-a_{\theta} l-\operatorname{Tr}\left(A_{\theta}(x, t) X\right)-b_{\theta}(x, t) p-f_{\theta}(x, t)\right\} \\
+\sum_{j=1}^{n} c_{i j}(x, t) r_{j},
\end{array}
$$


where $\Theta$ is compact, $a_{\theta}, A_{\theta}, b_{\theta}, f_{\theta}$ are bounded and continuous functions, $a_{\theta} \geq 0, A_{\theta}$ is positive (semi)definite, and $\left(c_{i j}\right)_{i j}$ satisfies the above conditions. This type of equation fits into assumption (F2); see Section 4.

The second type of problem we are interested in is related to models which are coercive in the gradient with the model form

$H_{i}(x, t, \mathbf{r}, p, X, l)=-l-\operatorname{Tr}(A(x, t) X)+b(x, t)|p|^{m}-f(x, t)+\sum_{j=1}^{n} c_{i j}(x, t) r_{j}$,

for some $m>1$ and $A, b, f$ are continuous and bounded, with $b$ uniformly positive, $A$ is positive (semi)definite, and taking $\left(c_{i j}\right)_{i j}$ as in the previous case. This type of nonlinearity arises in the context of Hamilton-Jacobi equations with unbounded controls and shall be considered as a particular example falling into assumption (F2)'.

In the last section of this article we construct classical sub and supersolutions verifying the initial condition (1.2). Using the comparison principle we get the existence of continuous viscosity solutions. As an application, we use the half-relaxed limits method introduced by Barles and Perthame [8] to conclude steady-state large time behavior for the solution of (1.1)-(1.2) when $c_{0}>0$ in (H2). Moreover, we find an exponential rate of convergence with a precise index given by $c_{0}$.

\section{Basic notation and viscosity evaluation}

In this brief chapter we introduce some notation associated with the viscosity formulation of our system. We represent by $B_{\delta}(x)$ the open ball centered at $x \in \mathbb{R}^{d}$ and radius $\delta>0$. If $x=0$, we write $B_{\delta}$ and $B$ if in addition $\delta=1$. Moreover, the open cylinder in the space-time is defined by

$$
\mathcal{C}_{\delta}(x, t):=B_{\delta}(x) \times(t-\delta, t+\delta) .
$$

For a nonempty set $A \subset \mathbb{R}^{d}$ and $x, p \in \mathbb{R}^{d}$ and $\phi: \mathbb{R}^{d} \rightarrow \mathbb{R}$, we also consider

$$
\mathcal{T}_{i}[A](\phi, x, t, p)=\int_{A}\left[\phi(x+z)-\phi(x)-\mathbb{1}_{B}\langle p, z\rangle\right] K_{i}(x, t, z) d z,
$$

and we omit the $p$-term in the case $p=D \phi(x)$, i.e., $\mathcal{T}_{i}[A](\phi, x, t)=$ $\mathcal{T}_{i}[A](\phi, x, t, D \phi(x))$.

To end this chapter we give a definition of viscosity solution to system (1.1)-(1.2), which is basically the one given in [23] and [48]. We state the definition in the finite horizon setting, for which we introduce the notation $Q_{T}=\mathbb{R}^{d} \times(0, T]$ for $T>0$. 
Definition 2.1. A bounded u.s.c. (resp. l.s.c.) function $\mathbf{u}: \bar{Q}_{T} \rightarrow \mathbb{R}^{n}$ is a viscosity subsolution (resp. supersolution) to problem (1.1)-(1.2) if $\mathbf{u}(\cdot, 0) \leq$ (resp. $\geq) \mathbf{u}_{0}$ in $\mathbb{R}^{d}$ and for each $\left(x_{0}, t_{0}\right) \in Q_{T}, i \in I(n)$, $\phi \in C^{2}\left(\bar{Q}_{T}\right)$, and $\delta>0$ such that $\left(x_{0}, t_{0}\right)$ is a maximum (resp. minimum) point to $u_{i}-\phi$ in $\mathcal{C}_{\delta}\left(x_{0}, t_{0}\right)$, then the following inequality holds:

$$
\begin{aligned}
\partial_{t} \phi\left(x_{0}, t_{0}\right)+H_{i}\left(x_{0}, t_{0}, \mathbf{u}\left(x_{0}, t_{0}\right), D \phi\left(x_{0}, t_{0}\right),\right. \\
\left.D^{2} \phi\left(x_{0}, t_{0}\right), \mathcal{T}_{i, \delta}\left(u_{i}, \phi, x_{0}, t_{0}\right)\right) \leq(\text { resp. } \geq) 0,
\end{aligned}
$$

where the last nonlocal dependence is understood as

$$
\mathcal{T}_{i, \delta}\left(u_{i}, \phi, x, t\right):=\mathcal{T}_{i}\left[B_{\delta}\right](\phi(\cdot, t), x, t)+\mathcal{T}_{i}\left[B_{\delta}^{c}\right]\left(u_{i}(\cdot, t), x, t, D \phi(x, t)\right) .
$$

Finally, $\mathbf{u}$ is a viscosity solution of (1.1)-(1.2), if $\mathbf{u}$ is a sub and supersolution of the system.

Following standard viscosity arguments the above definition can be equivalently stated by assuming that $u\left(x_{0}, t_{0}\right)=\phi\left(x_{0}, t_{0}\right)$ and that $\left(x_{0}, t_{0}\right)$ is a strict maximum point. Moreover, in order to avoid technicalities in the viscosity evaluation, the following lemmas will be useful in the sequel.

Lemma 2.2. Let $\mathbf{u}: \bar{Q}_{T} \rightarrow \mathbb{R}^{n}, \phi \in C^{2}\left(\bar{Q}_{T}\right), \delta>0$, and $i \in I(n)$. If $\left(x_{0}, t_{0}\right)$ is the maximum (resp. minimum) point of $u_{i}-\phi$ in $\mathcal{C}_{\delta}\left(x_{0}, t_{0}\right)$, then for all $0<\sigma \leq \delta$, we have

$$
\mathcal{T}_{i, \sigma}\left(u_{i}, \phi, x_{0}, t_{0}\right) \leq(\text { resp. } \geq) \mathcal{T}_{i, \delta}\left(u_{i}, \phi, x_{0}, t_{0}\right) .
$$

Proof: We focus on the "maximum" statement, hence

$$
u_{i}\left(x, t_{0}\right)-u_{i}\left(x_{0}, t_{0}\right) \leq \phi\left(x, t_{0}\right)-\phi\left(x_{0}, t_{0}\right) \quad \text { for each } x \in B_{\delta}\left(x_{0}\right) .
$$

Taking $\sigma \leq \delta$ and since the compensator term plays no role because it is common to both sides of the inequality, we see that

$$
\begin{aligned}
\mathcal{T}_{i, \sigma}\left(u_{i}, \phi, x_{0}, t_{0}\right)= & \mathcal{T}_{i}\left[B_{\delta}^{c}\right]\left(u_{i}\left(\cdot, t_{0}\right), x_{0}, t_{0}\right) \\
& +\mathcal{T}_{i}\left[B_{\delta} \backslash B_{\sigma}\right]\left(u_{i}\left(\cdot, t_{0}\right), x_{0}, t_{0}\right)+\mathcal{T}_{i}\left[B_{\sigma}\right]\left(\phi\left(\cdot, t_{0}\right), x_{0}, t_{0}\right) \\
\leq & \mathcal{T}_{i}\left[B_{\delta}^{c}\right]\left(u_{i}\left(\cdot, t_{0}\right), x_{0}, t_{0}\right) \\
& +\mathcal{T}_{i}\left[B_{\delta} \backslash B_{\sigma}\right]\left(\phi\left(\cdot, t_{0}\right), x_{0}, t_{0}\right)+\mathcal{T}_{i}\left[B_{\sigma}\right]\left(\phi\left(\cdot, t_{0}\right), x_{0}, t_{0}\right) \\
= & \mathcal{T}_{i, \delta}\left(u_{i}, \phi, x_{0}, t_{0}\right) .
\end{aligned}
$$

Lemma 2.3. Definition 2.1 can be equivalently formulated by taking $i \in$ $I(n)$, the test function $\phi \in C^{2}\left(\bar{Q}_{T}\right)$ bounded, and $\left(x_{0}, t_{0}\right)$ a global maximum (resp. minimum) point of $u_{i}-\phi$ in $\bar{Q}_{T}$ and instead of the viscosity inequality we write

$$
\begin{aligned}
\partial_{t} \phi\left(x_{0}, t_{0}\right)+H_{i}\left(x_{0}, t_{0}, \mathbf{u}\left(x_{0}, t_{0}\right), D \phi\left(x_{0}, t_{0}\right),\right. & \\
\left.D^{2} \phi\left(x_{0}, t_{0}\right), \mathcal{T}_{i} \phi\left(x_{0}, t_{0}\right)\right) & \leq(\text { resp. } \geq) 0 .
\end{aligned}
$$


Proof: By (H1), the general setting implies the new version. To prove the converse we focus only on the case of the subsolution. We consider $\phi \in C^{2}\left(\bar{Q}_{T}\right)$ such that $\left(x_{0}, t_{0}\right)$ is a strict maximum point for $u_{i}-\phi$ in $\mathcal{C}_{\delta}\left(x_{0}, t_{0}\right)$ with $\phi\left(x_{0}, t_{0}\right)=u_{i}\left(x_{0}, t_{0}\right)$. Then, there exists a sequence of functions $\left\{\tilde{\phi}_{n}\right\}$ bounded and smooth in $\bar{Q}_{T}$ such that $\tilde{\phi}_{n}=\phi$ in $\mathcal{C}_{\delta}\left(x_{0}, t_{0}\right)$, with $\tilde{\phi}_{n} \geq u_{i}$ in $\bar{Q}_{T}$ and such that $\tilde{\phi}_{n} \rightarrow u_{i}$ locally uniform in $\left(\bar{C}_{\delta}\left(x_{0}, t_{0}\right)\right)^{c}$. Then, since for each $n \in \mathbb{N},\left(x_{0}, t_{0}\right)$ is a global maximum point of $u_{i}-\tilde{\phi}_{n}$, we have

$$
\begin{aligned}
\partial_{t} \phi\left(x_{0}, t_{0}\right)+H_{i}\left(x_{0}, t_{0}, \mathbf{u}\left(x_{0}, t_{0}\right), D \phi\left(x_{0}, t_{0}\right),\right. \\
\left.D^{2} \phi\left(x_{0}, t_{0}\right), \mathcal{T}_{i} \tilde{\phi}_{n}\left(x_{0}, t_{0}\right)\right) \leq 0 .
\end{aligned}
$$

Applying the dominated convergence theorem, we can pass to the limit and get

$$
\mathcal{T}_{i}\left[B_{\delta}^{c}\right]\left(\tilde{\phi}_{n}\left(\cdot, t_{0}\right), x_{0}, t_{0}, D \phi\left(x_{0}, t_{0}\right)\right) \rightarrow \mathcal{T}_{i}\left[B_{\delta}^{c}\right]\left(u_{i}\left(\cdot, t_{0}\right), x_{0}, t_{0}, D \phi\left(x_{0}, t_{0}\right)\right),
$$

hence

$$
\mathcal{T}_{i} \tilde{\phi}_{n}\left(x_{0}, t_{0}\right) \rightarrow \mathcal{T}_{i, \delta}\left(u_{i}, \phi, x_{0}, t_{0}\right) .
$$

By the continuity of $H_{i}$, we conclude the desired inequality.

\section{Existence of discontinuous solutions}

Before stating our first results, we need to introduce extra notations. We write $\mathbf{u}^{*}$ for the upper semicontinuous envelope of $\mathbf{u}$, which is performed for each component, and similarly $\mathbf{u}_{*}$ for the lower semicontinuous envelope. Also, we give the following definition of discontinuous viscosity solution.

Definition 3.1. A bounded function $\mathbf{u}: \bar{Q}_{T} \rightarrow \mathbb{R}^{n}$ is a discontinuous viscosity subsolution (supersolution) of (1.1) if $\mathbf{u}^{*}$ is a subsolution $\left(\mathbf{u}_{*}\right.$ is a supersolution) of (1.1) in the sense of Definition 2.1. We say that $\mathbf{u}$ is a discontinuous viscosity solution to (1.1) if it is simultaneously a discontinuous sub and supersolution.

In what follows we perform Perron's method for nonlocal operators developed by Ishii in [24], adapted to systems where the diffusion terms are driven by variable-order nonlocal operators whose kernels depend on the space-time variable. Throughout this section we make assumptions (H1), (H2), and (K)-(S).

Theorem 3.2. Assume that $\mathbf{U} \in \operatorname{LSC}\left(\bar{Q}_{T}\right)$ and $\mathbf{V} \in \operatorname{USC}\left(\bar{Q}_{T}\right)$ are bounded discontinuous viscosity sub and supersolutions to problem (1.1) respectively. Then, there is a discontinuous viscosity solution $\mathbf{u}: \bar{Q}_{T} \rightarrow$ $\mathbb{R}^{n}$ to problem (1.1) with $\mathbf{U} \leq \mathbf{u} \leq \mathbf{V}$. 
Proof: We consider functions z: $\bar{Q}_{T} \rightarrow \mathbb{R}^{n}$ verifying the condition

$$
\mathbf{U} \leq \mathbf{z} \leq \mathbf{V} \quad \text { for all }(x, t) \in \bar{Q}_{T}
$$

and we set

$$
\Lambda=\left\{\mathbf{z}: \bar{Q}_{T} \rightarrow \mathbb{R}^{n} \mid \mathbf{z}^{*} \text { is a subsolution of (1.1) and verifies (3.1) }\right\} .
$$

We start by noticing that $\Lambda \neq \emptyset$ since $\mathbf{U}$ is a discontinuous viscosity subsolution and verifies (3.1). Hence, we define

$$
u_{i}(x, t)=\sup _{\mathbf{z} \in \Lambda} z_{i}(x, t), \quad \forall i \in I(n) .
$$

First we prove that $\mathbf{u}$ defined as above belongs to $\Lambda$. Indeed, it is direct to see that $\mathbf{u}$ verifies (3.1), and to prove that $\mathbf{u}$ is a viscosity subsolution we consider $i \in I(n), \delta>0,\left(x_{0}, t_{0}\right) \in Q_{T}$, and $\psi \in C^{2}\left(\bar{Q}_{T}\right)$ such that $u_{i}^{*}-\psi$ attains a strict maximum in $\left(x_{0}, t_{0}\right)$ at $\mathcal{C}_{\delta}\left(x_{0}, t_{0}\right)$.

By definition of $u_{i}^{*}$ and Lemma 2.10 of [19], there are sequences $\left(\mathbf{v}_{k}\right)_{k \in \mathbb{N}}$ in $\Lambda$ and $\left(y_{k}, t_{k}\right)_{k \in \mathbb{N}}$ in $\mathcal{C}_{\delta}\left(x_{0}, t_{0}\right)$, such that $\left(y_{k}, t_{k}\right) \rightarrow\left(x_{0}, t_{0}\right)$ and $\left(y_{k}, t_{k}\right)$ is a local maximum point of $v_{i, k}^{*}-\psi$. Moreover, $v_{i, k}^{*}\left(y_{k}, t_{k}\right) \rightarrow u_{i}^{*}\left(x_{0}, t_{0}\right)$. have

$$
\begin{aligned}
\partial_{t} \psi\left(y_{k}, t_{k}\right)+H_{i}\left(y_{k}, t_{k}, \mathbf{v}_{k}^{*}\left(y_{k}, t_{k}\right), D \psi\left(y_{k}, t_{k}\right),\right. \\
\left.D^{2} \psi\left(y_{k}, t_{k}\right), \mathcal{T}_{i, \delta}\left(v_{i, k}^{*}, \psi, y_{k}, t_{k}\right)\right) \leq 0 .
\end{aligned}
$$

Now we focus on the nonlocal operator. The continuity of $K_{i}$ and (K)-(S) imply

$$
\begin{aligned}
\int_{B_{\delta}}|z|^{2} K_{i}\left(y_{k}, t_{k}, z\right) d z & \leq \int_{B_{\delta}} \frac{|z|^{2}}{|z|^{d+2 \alpha_{i}\left(y_{k}, t_{k}, z\right)}} d z \\
& \leq \int_{B_{\delta}}|z|^{2\left(1-\alpha^{+}\right)-d} d z<+\infty
\end{aligned}
$$

and by the smoothness of $\psi$ it is possible to apply the dominated convergence theorem to conclude that

$$
\lim _{k \rightarrow \infty} \mathcal{T}_{i}\left[B_{\delta}\right]\left(\psi\left(\cdot, t_{k}\right), y_{k}, t_{k}\right)=\mathcal{T}_{i}\left[B_{\delta}\right]\left(\psi\left(\cdot, t_{0}\right), x_{0}, t_{0}\right)
$$


Also, using the definition of $\mathbf{u}$ we have that $u_{i}^{*} \geq v_{i, k}^{*}$ for all $k \in \mathbb{N}$, hence we can write

$$
\begin{aligned}
\mathcal{T}_{i}[ & \left.B_{\delta}^{c}\right]\left(v_{i, k}^{*}\left(\cdot, t_{k}\right), y_{k}, t_{k}, D \psi\left(y_{k}, t_{k}\right)\right) \\
= & {\left[u_{i}^{*}\left(x_{0}, t_{0}\right)-v_{i, k}^{*}\left(y_{k}, t_{k}\right)\right] \int_{B_{\delta}^{c}} K_{i}\left(y_{k}, t_{k}, z\right) d z } \\
& +\int_{B_{\delta}^{c}}\left[v_{i, k}^{*}\left(y_{k}+z, t_{k}\right)-u_{i}^{*}\left(x_{0}, t_{0}\right)-\mathbb{1}_{B}\left\langle D \psi\left(y_{k}, t_{k}\right), z\right\rangle\right] K_{i}\left(y_{k}, t_{k}, z\right) d z \\
\leq & o_{k}(1) \int_{B_{\delta}^{c}} K_{i}\left(y_{k}, t_{k}, z\right) d z+\int_{B_{\delta}^{c}}\left[u_{i}^{*}\left(y_{k}+z, t_{k}\right)-u_{i}^{*}\left(x_{0}, t_{0}\right)\right] K_{i}\left(y_{k}, t_{k}, z\right) d z \\
& -\int_{B \backslash B_{\delta}}\left\langle D \psi\left(y_{k}, t_{k}\right), z\right\rangle K_{i}\left(y_{k}, t_{k}, z\right) d z \\
:= & I_{1}+I_{2}+I_{3},
\end{aligned}
$$

where $o_{k}(1) \rightarrow 0$ as $k \rightarrow+\infty$.

To treat the term $I_{1}$, if $z \in B_{\delta}^{c}$, by $(\mathrm{K})$-(S) we notice that

$$
K_{i}\left(y_{k}, t_{k}, z\right) \leq C\left[\delta^{-2} \mathbb{1}_{B \backslash B_{\delta}}(z)|z|^{2\left(1-\alpha^{+}\right)-d}+\mathbb{1}_{B^{c}}(z)|z|^{-d-2 \alpha^{-}}\right],
$$

and since the last term is integrable in $B_{\delta}^{c}$, we have that $I_{1} \rightarrow 0$ as $k \rightarrow$ $+\infty$.

For the second term, since $u_{i}^{*}\left(y_{k}+z, t_{k}\right)-u_{i}^{*}\left(x_{0}, t_{0}\right) \geq-2|u|_{\infty}$, by $(3.5)$ and applying Fatou's lemma in its dominated version, we have that

$$
\limsup _{k \rightarrow+\infty} I_{2} \leq \int_{B_{\delta}^{c}}\left[u_{i}^{*}\left(x_{0}+z, t_{0}\right)-u_{i}^{*}\left(x_{0}, t_{0}\right)\right] K_{i}\left(x_{0}, t_{0}, z\right) d z .
$$

Finally, since $D \phi$ and $K_{i}$ are continuous, $\left(y_{k}, t_{k}\right) \rightarrow\left(x_{0}, t_{0}\right)$, and

$$
\left|\left\langle D \psi\left(y_{k}, t_{k}\right), z\right\rangle\right| K_{i}\left(y_{k}, t_{k}, z\right) \leq \frac{C}{\delta}|z|^{2\left(1-\alpha^{+}\right)-d},
$$

by the dominated convergence theorem we have that

$$
\lim _{k \rightarrow+\infty} I_{3}=-\int_{B \backslash B_{\delta}}\left\langle D \psi\left(x_{0}, t_{0}\right), z\right\rangle K_{i}\left(x_{0}, t_{0}, z\right) d z .
$$

Joining the previous calculations, we get that

$$
\begin{aligned}
\limsup _{k \rightarrow \infty} & \mathcal{T}_{i}\left[B_{\delta}^{c}\right]\left(v_{i, k}^{*}\left(\cdot, t_{k}\right), y_{k}, t_{k}, D \psi\left(y_{k}, t_{k}\right)\right) \\
& \leq \mathcal{T}_{i}\left[B_{\delta}^{c}\right]\left(u_{i}^{*}\left(\cdot, t_{0}\right), x_{0}, t_{0}, D \psi\left(x_{0}, t_{0}\right)\right) .
\end{aligned}
$$

Thus, by (3.4) and (3.6) we conclude that

$$
\limsup _{k \rightarrow+\infty} \mathcal{T}_{i, \delta}\left(v_{i, k}^{*}, \psi, y_{k}, t_{k}\right) \leq \mathcal{T}_{i, \delta}\left(u_{i}^{*}, \psi, x_{0}, t_{0}\right) .
$$


Now, from (3.3), (3.7), (H2), and the definition of $\mathbf{u}$, we notice that

$$
\begin{array}{r}
\partial_{t} \psi\left(y_{k}, t_{k}\right)+H_{i}\left(y_{k}, t_{k}, u_{1}^{*}, \ldots, v_{i, k}^{*}, \ldots, u_{n}^{*}, D \psi,\right. \\
\left.D^{2} \psi, \mathcal{T}_{i, \delta}\left(v_{i, k}^{*}, \psi, y_{k}, t_{k}\right)\right) \leq 0 .
\end{array}
$$

Now, taking $k \rightarrow+\infty$, we have

$$
\begin{aligned}
& \partial_{t} \psi\left(x_{0}, t_{0}\right)+H_{i}\left(x_{0}, t_{0}, \mathbf{u}^{*}\left(x_{0}, t_{0}\right), D \psi\left(x_{0}, t_{0}\right),\right. \\
&\left.D^{2} \psi\left(x_{0}, t_{0}\right), \limsup _{k \rightarrow+\infty} \mathcal{T}_{i, \delta}\left(v_{i, k}^{*}, \psi, y_{k}, t_{k}\right)\right) \leq 0 .
\end{aligned}
$$

Hence, by (3.7) and (H1) we have

$$
\partial_{t} \psi\left(x_{0}, t_{0}\right)+H_{i}\left(x_{0}, t_{0}, \mathbf{u}^{*}, D \psi, D^{2} \psi\left(x_{0}, t_{0}\right), \mathcal{T}_{i, \delta}\left(u_{i}^{*}, \psi, x_{0}, t_{0}\right)\right) \leq 0,
$$

and we conclude that $\mathbf{u}$ is a viscosity subsolution of (1.1).

In what follows we prove that the $\mathbf{u}_{*}$ given by $(3.2)$ is a viscosity supersolution of (1.1). By contradiction, we assume the existence of $i \in$ $I(n),\left(x_{0}, t_{0}\right) \in Q_{T}, \phi \in C^{2}\left(\bar{Q}_{T}\right)$ bounded such that $\left(x_{0}, t_{0}\right)$ is a strict global minimum point for $\left(u_{i}\right)_{*}-\phi$ on $\bar{Q}_{T}$ with $\left(u_{i}\right)_{*}\left(x_{0}, t_{0}\right)=\phi\left(x_{0}, t_{0}\right)$, and

$$
\begin{aligned}
& \partial_{t} \phi\left(x_{0}, t_{0}\right)+H_{i}\left(x_{0}, t_{0}, \mathbf{u}_{*}\left(x_{0}, t_{0}\right), D \phi\left(x_{0}, t_{0}\right),\right. \\
&\left.D^{2} \phi\left(x_{0}, t_{0}\right), \mathcal{T}_{i} \phi\left(x_{0}, t_{0}\right)\right)<-\theta
\end{aligned}
$$

for some $\theta>0$.

Since $\mathbf{V}$ is a supersolution to (1.1), (3.8) implies that $\left(u_{i}\right)_{*}\left(x_{0}, t_{0}\right)<$ $V_{i}\left(x_{0}, t_{0}\right)$. Then, by considering $r, \epsilon>0$ smaller if necessary we have

$$
\begin{aligned}
\phi(x, t)+\epsilon<V_{i}(x, t) & \text { for all }(x, t) \in C_{r}\left(x_{0}, t_{0}\right), \\
\phi(x, t)+\epsilon \leq\left(u_{i}\right)_{*}(x, t) & \text { for all }(x, t) \in C_{r_{0}}\left(x_{0}, t_{0}\right) \backslash C_{r}\left(x_{0}, t_{0}\right) .
\end{aligned}
$$

Moreover, by the smoothness and boundedness of $\phi$ and since the kernel $K_{i}$ is continuous and satisfies $(\mathrm{K})-(\mathrm{S})$, the function $(x, t) \mapsto \mathcal{T}_{i} \phi(x, t)$ is continuous. Using this, the continuity of $H_{i},(\mathrm{H} 2)$, the semicontinuity of $\mathbf{u}_{*}$, and in view of (3.8), for $r>0$ small in terms of $\theta$ we have

$$
\begin{aligned}
\partial_{t} \phi(x, t)+H_{i}\left(x, t,\left(\left(u_{1}\right)_{*}, \ldots, \phi, \ldots\right.\right. & \left.\left(u_{n}\right)_{*}\right)(x, t), D \phi(x, t), \\
& \left.D^{2} \phi(x, t), \mathcal{T}_{i} \phi(x, t)\right)<-\theta / 2
\end{aligned}
$$

for all $(x, t) \in C_{r}\left(x_{0}, t_{0}\right)$, where $\phi$ is in the $i$-th coordinate.

We consider the function $\mathbf{w}$ defined as $w_{j}=u_{j}$ for $j \neq i$ and

$$
w_{i}= \begin{cases}\max \left\{u_{i}, \phi+\epsilon\right\} & \text { in } \mathcal{C}_{r}\left(x_{0}, t_{0}\right), \\ u_{i} & \text { in } \mathcal{C}_{r}\left(x_{0}, t_{0}\right)^{c} .\end{cases}
$$

By the above considerations, since $\mathbf{U} \leq \mathbf{u} \leq \mathbf{V}$ in $\bar{Q}_{T}$ and $V_{i}>\phi+\epsilon$ in $C_{r}\left(x_{0}, t_{0}\right)$, then $\mathbf{w}$ verifies (3.1). In what follows, we prove that $\mathbf{w}^{*}$ is a viscosity subsolution to (1.1) and such that $w_{i}>u_{i}$ at some point in $\bar{Q}_{T}$ 
close to $\left(x_{0}, t_{0}\right)$. This contradicts the maximality of $\mathbf{u}$ and concludes the proof.

For this, we consider $j \in I(n),(\bar{x}, \bar{t}) \in Q_{T}, \varphi \in C^{2}\left(\bar{Q}_{T}\right)$, and $\sigma>0$ such that $(\bar{x}, \bar{t})$ is a strict maximum point of $w_{j}^{*}-\varphi$ in $C_{\sigma}(\bar{x}, \bar{t})$ with $w_{j}^{*}(\bar{x}, \bar{t})=\varphi(\bar{x}, \bar{t})$.

If $j \neq i$, by definition of $\mathbf{w}$ we have that $w_{j}=u_{j}$, and since $\mathbf{u}^{*}$ is a subsolution, we have that

$$
\partial_{t} \varphi(\bar{x}, \bar{t})+H_{j}\left(\bar{x}, \bar{t}, \mathbf{u}^{*}(\bar{x}, \bar{t}), D \varphi(\bar{x}, \bar{t}), D^{2} \varphi(\bar{x}, \bar{t}), \mathcal{T}_{j, \sigma}\left(u_{j}^{*}, \varphi, \bar{x}, \bar{t}\right)\right) \leq 0,
$$

moreover, since $w_{j}^{*}(\bar{x}, \bar{t})=u_{j}^{*}(\bar{x}, \bar{t})$ and $\mathbf{w}^{*} \geq \mathbf{u}^{*}$, we have that

$$
\mathcal{T}_{j, \sigma}\left(u_{j}^{*}, \varphi, \bar{x}, \bar{t}\right) \leq \mathcal{T}_{j, \sigma}\left(w_{j}^{*}, \varphi, \bar{x}, \bar{t}\right),
$$

hence, by (H1) and (H2), we have that

$$
\partial_{t} \varphi(\bar{x}, \bar{t})+H_{j}\left(\bar{x}, \bar{t}, \mathbf{w}^{*}(\bar{x}, \bar{t}), D \varphi(\bar{x}, \bar{t}), D^{2} \varphi(\bar{x}, \bar{t}), \mathcal{T}_{j, \sigma}\left(w_{j}^{*}, \varphi, \bar{x}, \bar{t}\right)\right) \leq 0 .
$$

Now, if $j=i$, we split the analysis into two cases: if $w_{i}^{*}(\bar{x}, \bar{t})=u_{i}^{*}(\bar{x}, \bar{t})$, similarly to the previous computations, we get the subsolution's viscosity inequality for $\mathbf{w}^{*}$ at $(\bar{x}, \bar{t})$.

When $w_{i}^{*}(\bar{x}, \bar{t})>u_{i}^{*}(\bar{x}, \bar{t})$, by $(3.9)$ we necessarily have that $(\bar{x}, \bar{t}) \in$ $C_{r}\left(x_{0}, t_{0}\right)$, hence, taking $\sigma^{\prime}>0$ such that $C_{\sigma^{\prime}}(\bar{x}, \bar{t}) \subset C_{r}\left(x_{0}, t_{0}\right)$, we consider

$$
\tilde{\phi}(x, t)= \begin{cases}\phi(x, t)+\epsilon & \text { in } \mathcal{C}_{\sigma^{\prime}}(\bar{x}, \bar{t}), \\ \phi(x, t) & \text { in } \mathcal{C}_{\sigma^{\prime}}(\bar{x}, \bar{t})^{c} .\end{cases}
$$

It is straightforward to see that

$$
\partial_{t} \tilde{\phi}(\bar{x}, \bar{t})=\partial_{t} \phi(\bar{x}, \bar{t}), \quad D \tilde{\phi}(\bar{x}, \bar{t})=D \phi(\bar{x}, \bar{t}), \quad D^{2} \tilde{\phi}(\bar{x}, \bar{t})=D^{2} \phi(\bar{x}, \bar{t}),
$$

and concerning the nonlocal terms it is direct to get from the definition of $\tilde{\phi}$ that

$$
\mathcal{T}_{i}\left[B_{\sigma^{\prime}}\right](\tilde{\phi}(\cdot, \bar{t}), \bar{x}, \bar{t})=\mathcal{T}_{i}\left[B_{\sigma^{\prime}}\right](\phi(\cdot, \bar{t}), \bar{x}, \bar{t}) .
$$

Now, by (3.5), we notice that

$$
\begin{aligned}
\mathcal{T}_{i}\left[B_{\sigma^{\prime}}^{c}\right](\tilde{\phi}(\cdot, \bar{t}), \bar{x}, \bar{t}) & =\mathcal{T}_{i}\left[B_{\sigma^{\prime}}^{c}\right](\phi(\cdot, \bar{t}), \bar{x}, \bar{t})-\epsilon \int_{B_{\sigma^{\prime}}^{c}} K_{i}(\bar{x}, \bar{t}, z) d z \\
& \geq \mathcal{T}_{i}\left[B_{\sigma^{\prime}}^{c}\right](\phi(\cdot, \bar{t}), \bar{x}, \bar{t})-C \epsilon\left(\sigma^{\prime}\right)^{-2} .
\end{aligned}
$$

Replacing this into (3.10) and using the continuity of $H_{i}$, taking $\epsilon$ small in terms of $\sigma^{\prime}$ and $\theta$ we get that $\tilde{\phi}$ satisfies the inequality

$$
\begin{array}{r}
\partial_{t} \tilde{\phi}(\bar{x}, \bar{t})+H_{i}\left(\bar{x}, \bar{t},\left(\left(u_{1}\right)_{*}, \ldots, \tilde{\phi}, \ldots\left(u_{n}\right)_{*}\right)(\bar{x}, \bar{t}), D \tilde{\phi}(\bar{x}, \bar{t}),\right. \\
\left.D^{2} \tilde{\phi}(\bar{x}, \bar{t}), \mathcal{T}_{i} \tilde{\phi}(\bar{x}, \bar{t})\right)<0 .
\end{array}
$$


Now, since $w_{i}^{*}(\bar{x}, \bar{t})>u_{i}^{*}(\bar{x}, \bar{t})$, we necessarily have that $w_{i}^{*}(\bar{x}, \bar{t})=\phi(\bar{x}, \bar{t})+$ $\epsilon$, and taking $0<\sigma^{\prime \prime}<\min \left(\sigma^{\prime}, \sigma\right)$ small enough we have that

$$
\varphi(x, t) \geq \phi(x, t)+\epsilon \quad \text { in } C_{\sigma^{\prime \prime}}(\bar{x}, \bar{t}), \quad \text { with } \varphi(\bar{x}, \bar{t})=\phi(\bar{x}, \bar{t})+\epsilon,
$$

and the smoothness of $\phi$ and $\varphi$ imply that

$$
\begin{gathered}
\partial_{t} \tilde{\phi}(\bar{x}, \bar{t})=\partial_{t} \varphi(\bar{x}, \bar{t}), \quad D \tilde{\phi}(\bar{x}, \bar{t})=D \varphi(\bar{x}, \bar{t}), \quad \text { and } \\
D^{2} \tilde{\phi}(\bar{x}, \bar{t}) \leq D^{2} \varphi(\bar{x}, \bar{t}) .
\end{gathered}
$$

From this we can get

$$
\mathcal{T}_{i}\left[B_{\sigma^{\prime \prime}}\right](\varphi(\cdot, \bar{t}), \bar{x}, \bar{t}) \geq \mathcal{T}_{i}\left[B_{\sigma^{\prime \prime}}\right](\tilde{\phi}(\cdot, \bar{t}), \bar{x}, \bar{t}),
$$

and since $w_{i}^{*} \geq \phi+\epsilon$ in $C_{\sigma^{\prime}}(\bar{x}, \bar{t})$, we have

$$
\begin{aligned}
\mathcal{T}_{i}\left[B_{\sigma^{\prime \prime}}^{c}\right]\left(w_{i}^{*}(\cdot, \bar{t}), \bar{x}, \bar{t}, D \varphi(\bar{x}, \bar{t})\right) \geq & \mathcal{T}_{i}\left[B_{\sigma^{\prime}}^{c}\right]\left(w_{i}^{*}(\cdot, \bar{t}), \bar{x}, \bar{t}, D \tilde{\phi}(\bar{x}, \bar{t})\right) \\
& +\mathcal{T}_{i}\left[B_{\sigma^{\prime}} \backslash B_{\sigma^{\prime \prime}}\right](\tilde{\phi}(\cdot, \bar{t}), \bar{x}, \bar{t}) .
\end{aligned}
$$

Adding the above inequalities, using that $w_{i}^{*} \geq \phi$ and the definition of $\tilde{\phi}$, we conclude that

$$
\mathcal{T}_{i, \sigma^{\prime \prime}}\left(w_{i}^{*}, \varphi, \bar{x}, \bar{t}\right) \geq \mathcal{T}_{i} \tilde{\phi}(\bar{x}, \bar{t}) .
$$

Then, using the last inequality, (3.11), (3.12), the properties (H1), (H2), and the definition of $\mathbf{w}$, we arrive at

$$
\partial_{t} \varphi(\bar{x}, \bar{t})+H_{i}\left(\bar{x}, \bar{t}, \mathbf{w}^{*}(\bar{x}, \bar{t}), D \varphi(\bar{x}, \bar{t}), D^{2} \varphi(\bar{x}, \bar{t}), \mathcal{T}_{i, \sigma^{\prime \prime}}\left(w_{i}^{*}, \varphi, \bar{x}, \bar{t}\right)\right)<0 .
$$

Finally, applying Lemma 2.2 and (H1), we obtain the viscosity inequality associated with $\sigma$.

\section{Comparison principle}

In order to state the comparison principle we present the main assumptions on the Hamiltonian $H$ and the kernel $K$, which are standard in the analysis of fully nonlinear equations.

In the first place, we state a regularity assumption of the Hamiltonian. (F1) $H(x, t, \mathbf{r}, p, X, l)$ is Lipschitz continuous in the nonlocal variable $l$, uniformly with respect to the other variables $(x, t, \mathbf{r}, p, X)$.

Also, we divide the proof of comparison into two main cases, mostly related with the behavior of the nonlinearity $H$ in terms of the gradient.

The first type is a linear gradient growth satisfying the following condition. 
(F2) For all $R>0$ and $T>0$, there exist moduli of continuity $\omega, \omega_{R, T}$ such that for all $|x|,|y| \leq R,|\mathbf{r}| \leq R, s, t \in[0, T], l \in \mathbb{R}$, and $X, Y$ satisfying the matrix inequality

$$
\left[\begin{array}{cc}
X & 0 \\
0 & -Y
\end{array}\right] \leq \gamma^{-1}\left[\begin{array}{cc}
I_{d} & -I_{d} \\
-I_{d} & I_{d}
\end{array}\right]+r(\beta)\left[\begin{array}{cc}
I_{d} & 0 \\
0 & I_{d}
\end{array}\right],
$$

for some $\gamma>0, r(\beta) \rightarrow 0$ as $\beta \rightarrow 0$. Then, if $o_{\beta}(1) \rightarrow 0$ as $\beta \rightarrow 0$, we have

$$
\begin{aligned}
H\left(y, t, \mathbf{r}, p+o_{\beta}(1), Y, l\right) & -H(x, s, \mathbf{r}, p, X, l) \\
\leq & \omega(\beta)+\omega_{R, T}((1+|p|)(|x-y|+|s-t|)),
\end{aligned}
$$

with $p=\gamma^{-1}(x-y)$.

The second type is superlinear Hamiltonians.

(F2)' There exist $m>1$ and $C>0$ such that, for all $R>0, T>0$, and all $\mu \in(0,1)$, there exist moduli of continuity $\omega, \omega_{R, T}$ such that for all $|x|,|y| \leq R,|\mathbf{r}| \leq R, t \in[0, T], l \in \mathbb{R}$, and $X, Y$ satisfying (4.1) for some $\gamma>0, r(\beta) \rightarrow 0$ as $\beta \rightarrow 0$. Then, if $o_{\beta}(1) \rightarrow 0$ as $\beta \rightarrow 0$, we have

$$
\begin{aligned}
& H\left(y, t, \mathbf{r}, p+o_{\beta}(1), Y, l\right)-\mu H\left(x, s, \mu^{-1} \mathbf{r}, \mu^{-1} p, \mu^{-1} X, \mu^{-1} l\right) \\
& \leq \omega_{R, T}(|x-y|+|s-t|)\left(1+|p|^{m}\right)+\omega(\beta)|p|^{m-1}+C(1-\mu)+(\mu-1)|p|^{m}, \\
& \text { with } p=\gamma^{-1}(x-y) .
\end{aligned}
$$

Moreover, we state a boundedness assumption of $H$.

(F3) For each $R>0$, there exists a constant $C_{H}(R)>0$ such that $|H(x, t, \mathbf{r}, p, X, l)| \leq C_{H}(R)$ for all $(x, t) \in Q$ and all $|\mathbf{r}|,|p|,|X|,|l| \leq$ $R$.

Finally, we consider the following integrability condition on the kernel. (K1) There exists a constant $C>0$, such that

$$
\int_{B}|K(x, t, z)-K(y, s, z)| d z \leq C(|x-y|+|t-s|) .
$$

Now we would like to give some examples of functions verifying hypothesis (K1). Note that this condition, for each $i \in I(n)$, complements hypothesis $(\mathrm{K})-(\mathrm{S})$, and it is a crucial technical integrability condition in the proof of the comparison principle.

We can see, as a simple example, that the kernel of the fractional Laplacian $K(x, t, z)=C|z|^{-d-2 \alpha}$ with constant $\alpha \in(0,1)$ verifies trivially $(\mathrm{K} 1)$. For more developed examples of functions that verify these conditions we can consider the following two cases: 


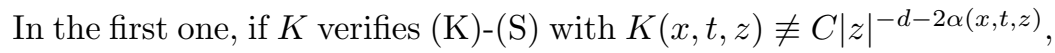
satisfying the assumption (K1) is enough to consider $K(x, t, z)$ locally Lipschitz in the variable $(x, t)$, uniformly with respect to the variable $z$.

On the other hand, if $K(x, t, z)=C|z|^{-d-2 \alpha(x, t, z)}$, to satisfy assumption (K1), we can consider for example

$$
|\alpha(x, t, z)-\alpha(y, s, z)| \leq C(z)(|x-y|+|t-s|)
$$

with $C(z) \leq|z|^{2\left(1+\alpha^{+}-\alpha^{-}\right)} /|\ln (|z|)|$ for all $z \in B \backslash\{0\}$ and $C(0)=0$.

At this moment, it is useful to consider the following technical results. Hence, we introduce some notation and we define

$$
I_{1}=\left\{j: H_{j} \text { satisfies }(\mathrm{F} 2)\right\} ; \quad I_{2}=\left\{j: H_{j} \text { satisfies (F2)' }\right\},
$$

and notice that $I(n)=I_{1} \cup I_{2}$ and the union is disjoint.

Lemma 4.1. Let $\mathbf{u}$ be a viscosity subsolution to system (1.1). For each $\eta>0$ and $\mu \in(0,1)$, the function $\mathbf{w}$ defined as

$$
w_{i}(x, t)=\mu_{i} u_{i}(x, t)-\eta t, \quad(x, t) \in \bar{Q}_{T}, i \in I(n),
$$

where

$$
\mu_{i}= \begin{cases}1 & \text { if } i \in I_{1}, \\ \mu & \text { if } i \in I_{2},\end{cases}
$$

is a viscosity subsolution to the system

$$
\begin{aligned}
\partial_{t} w_{i}+\mu_{i} H_{i}\left(x, t, \mu_{i}^{-1} \mathbf{w}, \mu_{i}^{-1} D w_{i},\right. \\
\left.\quad \mu_{i}^{-1} D^{2} w_{i}, \mu_{i}^{-1} \mathcal{T}_{i}\left(w_{i}\right)\right) \leq C(1-\mu)-\eta \quad \text { in } Q_{T},
\end{aligned}
$$

for some constant $C>0$ depending on $T,|\mathbf{u}|_{\infty}$, and $\left(c_{i j}\right)_{i, j \in I(n)}$ of (H2).

Proof: Let $i \in I(n), \delta>0,\left(x_{0}, t_{0}\right) \in Q_{T}$, and $\phi \in C^{2}\left(\bar{Q}_{T}\right)$ such that $w_{i}-\phi$ attains a maximum in $\left(x_{0}, t_{0}\right)$. We split our analysis into two cases:

If $i \in I_{1}$ and since $\mathbf{u}$ is a subsolution of (1.1), we have

$$
\partial_{t} \phi+H_{i}\left(x_{0}, t_{0}, \mathbf{u}, D \phi, D^{2} \phi, \mathcal{T}_{i, \delta}\left(w_{i}, \phi, x_{0}, t_{0}\right)\right) \leq-\eta \quad \text { in } Q_{T} .
$$

In the following computations, we drop the dependence of $H_{i}$ on $\left(x_{0}, t_{0}\right.$, $\left.D \phi, D^{2} \phi, \mathcal{T}_{i, \delta}\left(w_{i}, \phi, x_{0}, t_{0}\right)\right)$, since these variables do not play any role here. Also, we denote by $\left(\mathbf{e}_{i}\right)_{i \in I(n)}$ the canonical basis of $\mathbb{R}^{d}$.

Hence, taking $j \in I_{2}$, since $j \neq i$ by (H2), there exists $c_{i j} \leq 0$ such that

$$
H_{i}(\mathbf{u})-H_{i}\left(\mathbf{u}+\left(w_{j}-u_{j}\right) \mathbf{e}_{j}\right) \geq \begin{cases}0 & \text { if } u_{j} \leq w_{j}, \\ c_{i j}(1-\mu) u_{j}+c_{i j} \eta t & \text { if } u_{j} \geq w_{j}\end{cases}
$$

By (4.3) and taking $C_{j}=-c_{i j}|\mathbf{u}|_{\infty}>0$ we notice that

$$
\partial_{t} \phi+H_{i}\left(\mathbf{u}+\left(w_{j}-u_{j}\right) \mathbf{e}_{j}\right) \leq C_{j}(1-\mu)-c_{i j} \eta t-\eta .
$$


Now, if $j \in I_{1}$ (including the case $j=i$ ), we have that $w_{j}=u_{j}-\eta t \leq u_{j}$, and by $(\mathrm{H} 2)$

$$
H_{i}(\mathbf{u})-H_{i}\left(\mathbf{u}+\left(w_{j}-u_{j}\right) \mathbf{e}_{j}\right) \geq c_{i j} \eta t .
$$

Thus, by (4.3) there exists $C_{j}>0$ such that

$$
\partial_{t} \phi+H_{i}\left(\mathbf{u}+\left(w_{j}-u_{j}\right) \mathbf{e}_{j}\right) \leq-c_{i j} \eta t-\eta .
$$

By the above computations and iterating the procedure for each $j \in I(n)$, we conclude the desired viscosity formulation since

$$
\begin{aligned}
\partial_{t} \phi+H_{i}(\mathbf{w}) & \leq(1-\mu) \sum_{j \in I_{2}} C_{j}-\eta t \sum_{j \in I(n)} c_{i j}-\eta \\
& \leq C(1-\mu)-c_{0} \eta t-\eta \\
& \leq C(1-\mu)-\eta
\end{aligned}
$$

for a constant $C>0$ large enough and $c_{0} \geq 0$, the constant that appears in $(\mathrm{H} 2)$.

Now, if $i \in I_{2}$, using the fact that $\mathbf{u}$ is a subsolution of (1.1), we have

$$
\begin{aligned}
& \partial_{t} \phi+\mu H_{i}\left(x_{0}, t_{0}, \mathbf{u}, \mu^{-1} D \phi\right. \\
& \left.\mu^{-1} D^{2} \phi, \mu^{-1} \mathcal{T}_{i, \delta}\left(w_{i}, \phi, x_{0}, t_{0}\right)\right) \leq-\eta \quad \text { in } Q_{T} .
\end{aligned}
$$

Doing a similar analysis as in the previous case, for all $j \in I_{1}$ by (H2) we note that

$$
H_{i}(\mathbf{u})-H_{i}\left(\mathbf{u}+\left(\mu^{-1} w_{j}-u_{j}\right) \mathbf{e}_{j}\right) \geq \begin{cases}0 & \text { if } u_{j} \leq \mu^{-1} w_{j}, \\ c_{i j}\left(1-\mu^{-1}\right) u_{j}+c_{i j} \mu^{-1} \eta t & \text { if } u_{j} \geq \mu^{-1} w_{j} .\end{cases}
$$

By (4.4), there exists a constant $C_{j}>0$ such that

$$
\partial_{t} \phi+\mu H_{i}\left(\mathbf{u}+\left(\mu^{-1} w_{j}-u_{j}\right) \mathbf{e}_{j}\right) \leq C_{j}(1-\mu)-c_{i j} \eta t-\eta .
$$

Now, if $j \in I_{2}$ (including the case $j=i$ ), we have that $\mu^{-1} w_{j}=u_{j}-$ $\mu^{-1} \eta t \leq u_{j}$, and by $(\mathrm{H} 2)$

$$
H_{i}(\mathbf{u})-H_{i}\left(\mathbf{u}+\left(\mu^{-1} w_{j}-u_{j}\right) \mathbf{e}_{j}\right) \geq c_{i j} \mu^{-1} \eta t .
$$

Thus, by (4.4) there exists $C_{j}>0$ such that

$$
\partial_{t} \phi+\mu H_{i}\left(\mathbf{u}+\left(\mu^{-1} w_{j}-u_{j}\right) \mathbf{e}_{j}\right) \leq-c_{i j} \eta t-\eta .
$$

Iterating the procedure for each $j \in I(n)$, we conclude

$$
\begin{aligned}
\partial_{t} \phi+\mu H_{i}\left(\mu^{-1} \mathbf{w}\right) & \leq(1-\mu) \sum_{j \in I_{1}} C_{j}-\eta t \sum_{j \in I(n)} c_{i j}-\eta \\
& \leq C(1-\mu)-c_{0} \eta t-\eta \\
& \leq C(1-\mu)-\eta
\end{aligned}
$$

for a constant $C>0$ large enough. 
Before stating the comparison principle and following the spirit of [6], we introduce some properties of localization terms in the state variable.

Lemma 4.2. For each $i \in I(n)$, let $\mathcal{T}_{i}$ be defined as in (1.3). Let $\psi \in$ $C_{b}^{2}\left(\mathbb{R}^{d}\right)$. For $\beta>0$, define the function

$$
\psi_{\beta}(x)=\psi(\beta x), \quad x \in \mathbb{R}^{d} .
$$

Then, $\psi_{\beta}$ satisfies

$$
\left|D \psi_{\beta}\right|_{\infty} \leq C \beta, \quad\left|D^{2} \psi_{\beta}\right|_{\infty} \leq C \beta^{2}, \quad\left|\mathcal{T}_{i} \psi_{\beta}(\cdot, t)\right|_{\infty} \leq o_{\beta}(1),
$$

for all $t \in \mathbb{R}_{+}$, where $o_{\beta}(1) \rightarrow 0$ as $\beta \rightarrow 0$.

Proof: We concentrate on the nonlocal operator since the other results are direct. For each $(x, t) \in \mathbb{R}^{d} \times \mathbb{R}_{+}$, by $(\mathrm{K})$-(S) we have

$$
\begin{aligned}
&\left|\mathcal{T}_{i} \psi_{\beta}(x, t)\right| \leq\left|\mathcal{T}_{i}[B]\left(\psi_{\beta}, x, t\right)+\mathcal{T}_{i}\left[B_{\beta^{-1 / 2}} \mid B\right]\left(\psi_{\beta}, x, t\right)+\mathcal{T}_{i}\left[B_{\beta^{-1 / 2}}^{c}\right]\left(\psi_{\beta}, x, t\right)\right| \int_{B_{\beta^{-1 / 2} \backslash B}}|z| K_{i}(x, t, z) d z \\
& \leq C\left[\beta^{2} \int_{B}|z|^{2} K_{i}(x, t, z) d z+\beta\right.\left.+\int_{B_{\beta^{-1 / 2}}^{c}} K_{i}(x, t, z) d z\right] \\
& \leq C\left[\beta^{2} \int_{B}|z|^{-d+2\left(1-\alpha^{+}\right)} d z+\beta^{1 / 2} \int_{B_{\beta^{-1 / 2} \backslash B}}|z|^{-d-2 \alpha^{-}} d z\right. \\
&\left.\quad+\int_{B_{\beta^{-1 / 2}}^{c}}|z|^{-d-2 \alpha^{-}} d z\right] \\
& \leq C\left[\beta^{2}+\beta^{1 / 2}+\beta^{\alpha^{-}}\right] .
\end{aligned}
$$

At this moment we are able to establish the comparison principle for bounded viscosity sub and supersolutions.

Theorem 4.3. Assume (H1), (H2), (K)-(S), and that for each $i \in I(n)$ the kernel $K_{i}$ satisfies (K1) and the Hamiltonian $H_{i}$ satisfies (F1) and (F2), or (F2)'. Let $\mathbf{u}$ u.s.c. in $\bar{Q}_{T}$ be a bounded viscosity subsolution to (1.1), and $\mathbf{v}$ l.s.c. in $\bar{Q}_{T}$ be a bounded viscosity supersolution to (1.1) such that $\mathbf{u}(\cdot, 0) \leq \mathbf{v}(\cdot, 0)$ in $\mathbb{R}^{d}$. Then, $\mathbf{u} \leq \mathbf{v}$ in $\bar{Q}_{T}$.

Proof: We assume by contradiction that

$$
\max _{j \in I(n)} \sup _{(x, t) \in \bar{Q}_{T}}\left\{u_{j}(x, t)-v_{j}(x, t)\right\}:=\Theta>0 .
$$


We consider a function $\psi \in C_{b}^{2}\left(\mathbb{R}^{d}\right)$ with $\psi=0$ in $B_{1}, \psi \geq \max _{j \in I(n)} \sup _{\bar{Q}_{T}}\left\{u_{j}-\right.$ $\left.v_{j}\right\}+1$ in $B_{2}^{c}$, and for $\beta>0$ we define the function $\psi_{\beta}(x)=\psi(\beta x)$ for all $x \in \mathbb{R}^{d}$.

By taking $\beta, \eta>0$ small enough and $\mu$ close to 1 in terms of $T, \Theta$, $|\mathbf{u}|_{\infty},|\mathbf{v}|_{\infty}$, using the notation of Lemma 4.1 we consider $\mathbf{w}$ defined as in (4.2), hence we get

$$
\max _{j \in I(n)} \sup _{(x, t) \in \bar{Q}_{T}}\left\{w_{i}(x, t)-v_{i}(x, t)-\psi_{\beta}(x)\right\}:=\tilde{\Theta} \geq \Theta / 2>0 .
$$

At this point we start the standard process of doubling variables. For $0<\epsilon<\gamma$ we define

$$
\Psi(x, y, t, s, i):=w_{i}(x, s)-v_{i}(y, t)-\frac{|x-y|^{2}}{2 \gamma}-\frac{|t-s|^{2}}{2 \epsilon}-\psi_{\beta}(y)
$$

for all $(x, t),(y, s) \in \bar{Q}_{T}$ and $i \in I(n)$. Then, by (4.5) and the definition of $\Psi$ there exists a point $(\bar{x}, \bar{y}, \bar{s}, \bar{t}, \bar{i})$ such that

$$
\Psi(\bar{x}, \bar{y}, \bar{s}, \bar{t}, \bar{i})=\max _{j \in I(n)} \max _{(x, t) \in \bar{Q}_{T}} \Psi \geq \tilde{\Theta}>0 .
$$

Since $I(n)$ is finite, up to a subsequence if necessary, we can assume the index $\bar{i}$ above is fixed and independent of the other parameters, namely $i$.

Then, using the boundedness, the semicontinuity of $\mathbf{w}, \mathbf{v}$, and the maximal condition above we see that

(4.6) $|\bar{t}-\bar{s}|^{2} \leq C \epsilon, \quad|\bar{x}-\bar{y}|^{2} \leq C \gamma, \quad$ and $\quad \lim _{\gamma \rightarrow 0} \limsup _{\epsilon \rightarrow 0} \frac{|\bar{x}-\bar{y}|^{2}}{\gamma}=0$.

Moreover, the localization term $\psi_{\beta}$ makes $|\bar{x}|,|\bar{y}| \leq 4 / \beta$ for all $\gamma, \epsilon$ small enough. Then, up to a subsequence there exists $\left(x^{*}, t^{*}\right) \in \bar{Q}_{T}$ such that $\bar{t}, \bar{s} \rightarrow t^{*}$ and $\bar{x}, \bar{y} \rightarrow x^{*}$ as $\epsilon, \gamma \rightarrow 0$. And using that $\mathbf{u} \leq \mathbf{v}$ at $t=0$ it is possible to conclude that $\bar{s}, \bar{t}>0$ uniformly in $\epsilon, \gamma$ when $\beta>0$ is fixed small and $\mu<1$ is fixed close to 1 in terms of $\tilde{\Theta}$.

Now, in order to establish the viscosity evaluations, we denote

$$
\phi_{1}(x, s)=v_{i}(\bar{y}, \bar{t})+\frac{|x-\bar{y}|^{2}}{2 \gamma}+\frac{|s-\bar{t}|^{2}}{2 \epsilon}+\psi_{\beta}(\bar{y}),
$$

which serves as a test function for $\mathbf{w}$ at $(\bar{x}, \bar{s})$, and

$$
\phi_{2}(y, t)=w_{i}(\bar{x}, \bar{s})-\frac{|\bar{x}-y|^{2}}{2 \gamma}-\frac{|\bar{s}-t|^{2}}{2 \epsilon}-\psi_{\beta}(y),
$$

which serves as a test function for $v_{i}$ at $(\bar{y}, \bar{t})$. Thus, applying the Crandall-Ishii-Lions lemma for nonlocal problems given by Corollary 1 in [6], 
properly adapted to our current framework, and by Lemma 4.1 we can write

$$
\begin{aligned}
& \partial_{t} \phi_{1}(\bar{x}, \bar{s})+\mu_{i} H_{i}\left(\bar{x}, \bar{s}, \mu_{i}^{-1} \mathbf{w}, \mu_{i}^{-1} \bar{p}, \mu_{i}^{-1} X, \mu_{i}^{-1} \mathcal{T}_{i, \delta}\left(w_{i}, \phi_{1}, \bar{x}, \bar{s}\right)\right) \leq C(1-\mu)-\eta \\
& \leq C\left(\bar{y}, \bar{t}, \bar{t}, \bar{v}, Y, \mathcal{T}_{i, \delta}\left(v_{i}, \phi_{2}, \bar{y}, \bar{t}\right)\right) \\
& \partial_{t} \phi_{2}(\bar{y}, \bar{t})+H_{i}(\bar{y}, \bar{t}
\end{aligned}
$$

where $\bar{p}=\gamma^{-1}(\bar{x}-\bar{y}), \bar{q}=\bar{p}-D \psi_{\beta}(\bar{y})$, and $X, Y$ are symmetric matrices satisfying condition (4.1), for any $\delta>0$ small enough.

Taking into account that $\partial_{t} \phi_{1}(\bar{x}, \bar{s})=\partial_{t} \phi_{2}(\bar{y}, \bar{t})$ and subtracting the above equations we obtain that

$$
\begin{aligned}
& H_{i}\left(\bar{y}, \bar{t}, \mathbf{v}, \bar{q}, Y, \mathcal{T}_{i, \delta}\left(v_{i}, \phi_{2}, \bar{y}, \bar{t}\right)\right) \\
& \quad-\mu_{i} H_{i}\left(\bar{x}, \bar{s}, \mu_{i}^{-1} \mathbf{w}, \mu_{i}^{-1} \bar{p}, \mu_{i}^{-1} X, \mu_{i}^{-1} \mathcal{T}_{i, \delta}\left(w_{i}, \phi_{1}, \bar{x}, \bar{s}\right)\right) \geq \eta-C(1-\mu) .
\end{aligned}
$$

Calling the first term of the above inequality $A$, we have

$$
\begin{aligned}
A= & H_{i}\left(\bar{y}, \bar{t}, \mathbf{v}(\bar{y}, \bar{t}), \bar{q}, Y, \mathcal{T}_{i, \delta}\left(v_{i}, \phi_{2}, \bar{y}, \bar{t}\right)\right) \\
& -\mu_{i} H_{i}\left(\bar{x}, \bar{s}, \mu_{i}^{-1} \mathbf{v}(\bar{y}, \bar{t}), \mu_{i}^{-1} \bar{p}, \mu_{i}^{-1} X, \mu_{i}^{-1} \mathcal{T}_{i, \delta}\left(v_{i}, \phi_{2}, \bar{y}, \bar{t}\right)\right) \\
+ & \mu_{i}\left[H_{i}\left(\bar{x}, \bar{s}, \mu_{i}^{-1} \mathbf{v}(\bar{y}, \bar{t}), \mu_{i}^{-1} \bar{p}, \mu_{i}^{-1} X, \mu_{i}^{-1} \mathcal{T}_{i, \delta}\left(v_{i}, \phi_{2}, \bar{y}, \bar{t}\right)\right)\right. \\
& \left.\quad-H_{i}\left(\bar{x}, \bar{s}, \mu_{i}^{-1} \mathbf{v}(\bar{y}, \bar{t}), \mu_{i}^{-1} \bar{p}, \mu_{i}^{-1} X, \mu_{i}^{-1} \mathcal{T}_{i, \delta}\left(w_{i}, \phi_{1}, \bar{x}, \bar{s}\right)\right)\right] \\
+ & \mu_{i}\left[H_{i}\left(\bar{x}, \bar{s}, \mu_{i}^{-1} \mathbf{v}(\bar{y}, \bar{t}), \mu_{i}^{-1} \bar{p}, \mu_{i}^{-1} X, \mu_{i}^{-1} \mathcal{T}_{i, \delta}\left(w_{i}, \phi_{1}, \bar{x}, \bar{s}\right)\right)\right. \\
& \left.\quad-H_{i}\left(\bar{x}, \bar{s}, \mu_{i}^{-1} \mathbf{w}(\bar{x}, \bar{s}), \mu_{i}^{-1} \bar{p}, \mu_{i}^{-1} X, \mu_{i}^{-1} \mathcal{T}_{i, \delta}\left(w_{i}, \phi_{1}, \bar{x}, \bar{s}\right)\right)\right] \\
:= & A_{1}+A_{2}+A_{3} .
\end{aligned}
$$

Now, by Lemma 4.2 we have that $\bar{q}=\bar{p}+o_{\beta}(1)$ with $o_{\beta}(1) \rightarrow 0$ as $\beta \rightarrow 0$. Hence, to estimate $B_{1}$ we divide the analysis into cases: if $H_{i}$ satisfies (F2), then $\mu_{i}=1$, hence by applying (F2) directly we see that

$$
A_{1} \leq o_{\beta}(1)+\omega_{\beta, T}((1+|\bar{p}|)(|\bar{x}-\bar{y}|+|\bar{s}-\bar{t}|)) .
$$

If $H_{i}$ satisfies (F2)', then $\mu_{*}=\mu$ and we see that

$A_{1} \leq \omega_{\beta, T}(|\bar{x}-\bar{y}|+|\bar{s}-\bar{t}|)\left(1+|\bar{p}|^{m}\right)+o_{\beta}(1)|\bar{p}|^{m-1}+C(1-\mu)+(\mu-1)|\bar{p}|^{m}$.

Then, if $\bar{p}$ is uniformly bounded when $\gamma$ is small, it is easy to see that

$$
A_{1} \leq C \omega_{\beta, T}(|\bar{x}-\bar{y}|+|\bar{s}-\bar{t}|)+o_{\beta}(1)+C(1-\mu) .
$$

Otherwise, if $\bar{p}$ is unbounded, for $\mu<1$ fixed, we consider $\epsilon \ll \gamma$ and for all $\gamma, \beta$ small enough in terms of $1-\mu$, such that (4.6) implies

$$
\begin{aligned}
A_{1} \leq & \omega_{\beta, T}(|\bar{x}-\bar{y}|+|\bar{s}-\bar{t}|)+C(1-\mu) \\
& +\left[\omega_{\beta, T}(|\bar{x}-\bar{y}|+|\bar{s}-\bar{t}|)+o_{\beta}(1)|\bar{p}|^{-1}+(\mu-1)\right]|\bar{p}|^{m} \\
\leq & \omega_{\beta, T}(|\bar{x}-\bar{y}|+|\bar{s}-\bar{t}|)+C(1-\mu) .
\end{aligned}
$$


Then, summarizing the above estimates and considering $\epsilon, \gamma, \beta$ as above, by (4.6) we conclude

$$
A_{1} \leq o_{\gamma}(1)+o_{\beta}(1)+C(1-\mu) .
$$

Now, before dealing with the term $A_{2}$, we analyze the nonlocal operators. Hence, by definition of $\phi_{2}$ and $(\mathrm{K})-(\mathrm{S})$, we have

$$
\begin{aligned}
\left|\mathcal{T}_{i}\left[B_{\delta}\right]\left(\phi_{2}(\cdot, \bar{t}), \bar{y}\right)-\mathcal{T}_{i}\left[B_{\delta}\right]\left(\psi_{\beta}, \bar{y}, t\right)\right| & \leq C \gamma^{-1} \int_{B_{\delta}}|z|^{2} K(\bar{y}, \bar{t}, z) d z \\
& \leq C \gamma^{-1} \int_{B_{\delta}}|z|^{-d+2\left(1-\alpha^{+}\right)} d z
\end{aligned}
$$

then,

$$
\mathcal{T}_{i}\left[B_{\delta}\right]\left(\phi_{2}(\cdot, \bar{t}), \bar{y}\right)=\gamma^{-1} o_{\delta}(1)-\mathcal{T}_{i}\left[B_{\delta}\right]\left(\psi_{\beta}, \bar{y}, t\right)
$$

Similarly,

$$
\mathcal{T}_{i}\left[B_{\delta}\right]\left(\phi_{1}(\cdot, \bar{s}), \bar{x}\right)=\gamma^{-1} o_{\delta}(1)
$$

Moreover, by the maximality of $(\bar{x}, \bar{y}, \bar{s}, \bar{t})$ we see that

$$
v_{i}(\bar{y}+z, \bar{t})-v_{i}(\bar{y}, \bar{t}) \geq w_{i}(\bar{x}+z, \bar{s})-w_{i}(\bar{x}, \bar{s})-\psi_{\beta}(\bar{y}+z)+\psi_{\beta}(\bar{y}),
$$

hence

$$
\begin{aligned}
& \mathcal{T}_{i}\left[B_{\delta}^{c}\right]\left(v_{i}(\cdot, \bar{t}), \bar{y}, \bar{q}\right) \\
& \geq \int_{B_{\delta}^{c}}\left[w_{i}(\bar{x}+z, \bar{s})-w_{i}(\bar{x}, \bar{s})-\mathbb{1}_{B}(z)\langle\bar{p}, z\rangle\right] K_{i}(\bar{y}, \bar{t}, z) d z-\mathcal{T}_{i}\left[B_{\delta}^{c}\right]\left(\psi_{\beta}, \bar{y}, \bar{t}\right) \\
& =\mathcal{T}_{i}\left[B_{\delta}^{c}\right]\left(w_{i}(\cdot, \bar{s}), \bar{x}, \bar{p}\right)+\mathcal{I}\left(w_{i}, \bar{x}, \bar{s}, \bar{y}, \bar{t}\right)-\mathcal{T}_{i}\left[B_{\delta}^{c}\right]\left(\psi_{\beta}, \bar{y}, \bar{t}\right) \mid
\end{aligned}
$$

where

$$
\begin{aligned}
& \mathcal{I}\left(w_{i}, \bar{x}, \bar{s}, \bar{y}, \bar{t}\right) \\
& :=\int_{B_{\delta}^{c}}\left[w_{i}(\bar{x}+z, \bar{s})-w_{i}(\bar{x}, \bar{s})-\mathbb{1}_{B}(z)\langle\bar{p}, z\rangle\right]\left[K_{i}(\bar{y}, \bar{t}, z)-K_{i}(\bar{x}, \bar{s}, z)\right] d z
\end{aligned}
$$

Now we concentrate to get a bound for the term $\mathcal{I}$, hence

$$
\begin{aligned}
\mathcal{I}\left(w_{i}, \bar{x}, \bar{s}, \bar{y}, \bar{t}\right)= & \int_{B \backslash B_{\delta}}\left[w_{i}(\bar{x}+z, \bar{s})-w_{i}(\bar{x}, \bar{s})\right]\left[K_{i}(\bar{y}, \bar{t}, z)-K_{i}(\bar{x}, \bar{s}, z)\right] d z \\
& -\int_{B \backslash B_{\delta}}\langle\bar{p}, z\rangle\left[K_{i}(\bar{y}, \bar{t}, z)-K_{i}(\bar{x}, \bar{s}, z)\right] d z \\
& +\int_{B^{c}}\left[w_{i}(\bar{x}+z, \bar{s})-w_{i}(\bar{x}, \bar{s})\right]\left[K_{i}(\bar{y}, \bar{t}, z)-K_{i}(\bar{x}, \bar{s}, z)\right] d z \\
:= & I_{1}+I_{2}+I_{3} .
\end{aligned}
$$


For the terms $I_{1}$ and $I_{2}$, by (K1) we notice that

$$
\left|I_{1}\right| \leq 2|u|_{\infty} \int_{B}\left|K_{i}(\bar{y}, \bar{t}, z)-K_{i}(\bar{x}, \bar{s}, z)\right| d z \leq C(|\bar{x}-\bar{y}|+|\bar{t}-\bar{s}|),
$$

and

$$
\left|I_{2}\right| \leq \gamma^{-1}|\bar{x}-\bar{y}| \int_{B}\left|K_{i}(\bar{y}, \bar{t}, z)-K_{i}(\bar{x}, \bar{s}, z)\right| d z \leq C \frac{|\bar{x}-\bar{y}|}{\gamma}(|\bar{x}-\bar{y}|+|\bar{t}-\bar{s}|) .
$$

Now, since $K_{i}$ is continuous, $\bar{t}, \bar{s} \rightarrow t^{*}$ and $\bar{x}, \bar{y} \rightarrow x^{*}$ as $\epsilon, \gamma \rightarrow 0$, we have that $\left|K_{i}(\bar{y}, \bar{t}, z)-K_{i}(\bar{x}, \bar{s}, z)\right| \rightarrow 0$. Moreover, by $(\mathrm{K})$-(S) we get

$$
\left|K_{i}(\bar{y}, \bar{t}, z)-K_{i}(\bar{x}, \bar{s}, z)\right| \leq 2 C|z|^{-d-2 \alpha^{-}} \quad \text { if } z \in B^{c} .
$$

Hence, by the dominated convergence theorem we have that

$$
\left|I_{3}\right| \leq 2|u|_{\infty} \int_{B^{c}}\left|K_{i}(\bar{y}, \bar{t}, z)-K_{i}(\bar{x}, \bar{s}, z)\right| d z=o_{\gamma}(1) .
$$

By the above computations and (4.6), we obtain that $\mathcal{T}_{i}\left(\bar{w}_{i}, \bar{x}, \bar{s}, \bar{y}, \bar{t}\right)=$ $o_{\gamma}(1)$ as $\varepsilon \ll \gamma$ and $\gamma \rightarrow 0$.

Hence, if we join the approach of $\mathcal{I}$ with (4.7), (4.8), and (4.9), by Lemma 4.2 we get that

$$
\mathcal{T}_{i, \delta}\left(v_{i}, \phi_{2}, \bar{y}, \bar{t}\right) \geq \mathcal{T}_{i, \delta}\left(w_{i}, \phi_{1}, \bar{x}, \bar{s}\right)+o_{\beta}(1)+o_{\gamma}(1)+\gamma^{-1} o_{\delta}(1) .
$$

Now, by (4.10), (H1), and (F1), we have

$$
\begin{aligned}
& A_{2} \leq \mu_{i}\left[H _ { i } \left(\bar{x}, \bar{s}, \mu_{i}^{-1} \mathbf{v}(\bar{y}, \bar{t}), \mu_{i}^{-1} \bar{p}, \mu_{i}^{-1} X, \mu_{i}^{-1}\left(\mathcal{T}_{i, \delta}\left(w_{i}, \phi_{1}, \bar{x}, \bar{s}\right)+o_{\beta}(1)\right.\right.\right.\left.\left.+o_{\gamma}(1)+\gamma^{-1} o_{\delta}(1)\right)\right) \\
&\left.-H_{i}\left(\bar{x}, \bar{s}, \mu_{i}^{-1} \mathbf{v}(\bar{y}, \bar{t}), \mu_{i}^{-1} \bar{p}, \mu_{i}^{-1} X, \mu_{i}^{-1} \mathcal{T}_{i, \delta}\left(w_{i}, \phi_{1}, \bar{x}, \bar{s}\right)\right)\right] \\
& \leq o_{\beta}(1)+o_{\gamma}(1)+\gamma^{-1} o_{\delta}(1) .
\end{aligned}
$$

To deal with the last term we use the monotone property (H2). Then, we define

$$
\begin{aligned}
G_{i}(k):=H_{i}\left(\bar{x}, \bar{s}, v_{1}(\bar{y}, \bar{t}), \ldots, v_{k}(\bar{y}, \bar{t}), w_{k+1}(\bar{x}, \bar{s}), \ldots,\right. \\
\left.w_{n}(\bar{x}, \bar{s}), \mu_{i}^{-1} \bar{p}, \mu_{i}^{-1} X, \mu_{i}^{-1} \mathcal{T}_{i, \delta}\left(w_{i}, \phi_{1}, \bar{x}, \bar{s}\right)\right),
\end{aligned}
$$

where $G_{i}(0)=H_{i}\left(\bar{x}, \bar{s}, \mathbf{w}(\bar{x}, \bar{s}), \mu_{i}^{-1} \bar{p}, \mu_{i}^{-1} X, \mu_{i}^{-1} \mathcal{T}_{i, \delta}\left(w_{i}, \phi_{1}, \bar{x}, \bar{s}\right)\right)$. Hence,

$$
A_{3}=-\mu_{i} \sum_{k=1}^{n}\left[G_{i}(k-1)-G_{i}(k)\right]
$$


By definition of $\Psi, \mathbf{w}$, and $\mathbf{v}$, we have that

$$
\begin{aligned}
& w_{i}(\bar{x}, \bar{s})-v_{i}(\bar{y}, \bar{t}) \geq \tilde{\Theta} \quad \text { and } \\
& w_{i}(\bar{x}, \bar{s})-v_{i}(\bar{y}, \bar{t}) \geq w_{k}(\bar{x}, \bar{s})-v_{k}(\bar{y}, \bar{t})
\end{aligned} \text { for } \quad k \neq i,
$$

therefore, using (H2) for all $k \neq i$ we obtain

$$
G_{i}(k-1)-G_{i}(k) \geq \begin{cases}0 & \text { if } w_{k}(\bar{x}, \bar{s}) \leq v_{k}(\bar{y}, \bar{t}), \\ c_{i k}\left(w_{k}(\bar{x}, \bar{s})-v_{k}(\bar{y}, \bar{t})\right) & \text { if } w_{k}(\bar{x}, \bar{s}) \geq v_{k}(\bar{y}, \bar{t}),\end{cases}
$$

and since $c_{i k} \leq 0$ for all $k \neq i$, by (4.11) we have

$$
G_{i}(k-1)-G_{i}(k) \geq c_{i k}\left(w_{i}(\bar{x}, \bar{s})-v_{i}(\bar{y}, \bar{t})\right) .
$$

Again, by (H2) and (4.11) we have

$$
G_{i}(i-1)-G_{i}(i) \geq c_{i i}\left(w_{i}(\bar{x}, \bar{s})-v_{i}(\bar{y}, \bar{t})\right) .
$$

Then, we have

$$
A_{3} \leq-\mu_{i}\left(w_{i}(\bar{x}, \bar{s})-v_{i}(\bar{y}, \bar{t})\right) \sum_{k=1}^{n} c_{i k} \leq-c_{0} \mu \tilde{\Theta} .
$$

Finally, replacing the bounds of $A$ in (4.7), we obtain

$$
0<\eta+c_{0} \mu \tilde{\Theta} \leq \gamma^{-1} o_{\delta}(1)+o_{\gamma}(1)+o_{\beta}(1)+C(1-\mu) .
$$

Fixing $\eta>0$ and letting $\delta, \epsilon, \gamma, \beta \rightarrow 0$ and $\mu \rightarrow 1$, we arrive at a contradiction. This concludes the proof.

\section{Well-posedness and large time behavior}

The main result of this paper is the following existence and uniqueness for system (1.1)-(1.2).

Theorem 5.1. Assume the hypotheses of Theorem 4.3 and that for each $i \in I(n)$ the Hamiltonian $H_{i}$ satisfies (F3). Then, for the initial data $\mathbf{u}_{0} \in C_{b}\left(\mathbb{R}^{d}, \mathbb{R}^{n}\right)$, there exists a unique viscosity solution $\mathbf{u} \in C(\bar{Q})$ for the Cauchy problem (1.1)-(1.2).

Moreover, if $c_{0}>0$ in (H2), the solution $\mathbf{u}$ is uniformly bounded and we have the estimate

$$
\left|u_{i}(x, t)\right| \leq C+\left|\mathbf{u}_{0}\right|_{\infty}, \quad \text { for all }(x, t) \in Q, i \in I(n),
$$

for some constant $C>0$ depending on $c_{0}$.

Proof: We begin by considering $Q_{T}$ with $T>0$. First we assume that $u_{0, i} \in C_{b}^{2}\left(\mathbb{R}^{d}\right)$ for all $i \in I(n)$, with $\left|\mathbf{u}_{0}\right|_{C^{2}\left(\mathbb{R}^{d}, \mathbb{R}^{n}\right)}<+\infty$. Then, we consider $V_{i}(x, t)=C t+u_{0, i}(x)$ for some $C>0$ to be fixed later, for all $i \in I(n)$.

By $(\mathrm{K})-(\mathrm{S})$ we see that

$$
\left|\mathcal{T}_{i} u_{0, i}(x, t)\right| \leq R
$$


for some $R>0$ fixed. By (F3) and for each $i \in I(n)$, there exists a constant $C_{H, i}(R)>0$ such that

$$
\begin{aligned}
\partial_{t} V_{i}(x, t) & +H_{i}\left(x, t, \mathbf{V}, D V_{i}, D^{2} V_{i}, \mathcal{T}_{i} V_{i}(x, t)\right) \\
\geq & C+H_{i}\left(x, t, \mathbf{u}_{0}, D u_{0, i}, D^{2} u_{0, i}, \mathcal{T}_{i} u_{0, i}(x, t)\right) \\
\geq & C-C_{H, i}(R)
\end{aligned}
$$

for all $(x, t) \in Q_{T}$. Taking $C>0$ large in terms of $\max _{i \in I(n)}\left\{C_{H, i}(R)\right\}$, we thus get that $\mathbf{V}$ is a classical supersolution to (1.1) for the problem set up on $Q_{T}$, which satisfies $\mathbf{V}(x, 0)=\mathbf{u}_{0}(x)$ for all $x \in \mathbb{R}^{d}$.

Similarly, the function $U_{i}(x, t)=-C t+u_{0, i}(x)$ for all $i \in I(n)$ is a classical subsolution to (1.1) for some $C>0$ large enough verifying $\mathbf{U}(x, 0)=\mathbf{u}_{0}(x)$. Hence, Theorem 3.2 implies the existence of a discontinuous viscosity solution $\mathbf{u}$ for (1.1) such that $\mathbf{u}^{*}(x, 0)=\mathbf{u}_{*}(x, 0)=\mathbf{u}_{0}(x)$ for all $x \in \mathbb{R}^{d}$ and thus by Theorem 4.3 we conclude $\mathbf{u} \in C\left(\bar{Q}_{T}\right)$. Uniqueness follows again by Theorem 4.3 .

In the general case, when $\mathbf{u}_{0} \in C_{b}\left(\mathbb{R}^{d}, \mathbb{R}^{n}\right)$, we take a sequence $\left(\mathbf{u}_{0}^{\epsilon}\right)_{\epsilon}$ in $C_{b}^{2}\left(\mathbb{R}^{d}, \mathbb{R}^{n}\right)$ with $\left|\mathbf{u}_{0}^{\epsilon}\right|_{C^{2}\left(\mathbb{R}^{d}, \mathbb{R}^{n}\right)}<+\infty$ and $\left|\mathbf{u}_{0}^{\epsilon}-\mathbf{u}_{0}\right|_{\infty}<\epsilon$ for all $\epsilon>0$. Thus, by the previous analysis we can construct a supersolution $V_{i}^{\epsilon}(x, t)=u_{0, i}^{\epsilon}(x)+\epsilon+C_{\epsilon} t$, and a subsolution $U_{i}^{\epsilon}(x, t)=u_{0, i}^{\epsilon}(x)-\epsilon-C_{\epsilon} t$ to equation (1.1). Then, defining

$U_{i}(x, t)=\sup _{\epsilon>0}\left\{U_{i}^{\epsilon}(x, t)\right\} \quad$ and $\quad V_{i}(x, t)=\inf _{\epsilon>0}\left\{V_{i}^{\epsilon}(x, t)\right\} \quad$ for all $i \in I(n)$,

by the first part of the proof of Theorem 3.2 we have that $\mathbf{U}, \mathbf{V}$ are respectively viscosity sub and supersolutions to problem (1.1) and match with $\mathbf{u}_{0}$ at $t=0$. From this point we follow the same lines of the previous case.

Finally, given $0<T<T^{\prime}$, the viscosity solution in $\left(0, T^{\prime}\right]$ must coincide in $(0, T]$ with the viscosity solution in this interval, by uniqueness. Thus, the viscosity solution of (1.1)-(1.2) extends uniquely to all $t \in$ $[0,+\infty)$, i.e., it is global in time.

For the estimate (5.1), we consider the constant function $V_{i}=C+$ $\left|\mathbf{u}_{0}\right|_{\infty}>0$ for all $i \in I(n)$, hence by (H2) for each $j \in I(n)$ we have

$$
\begin{array}{r}
H_{i}\left(x, t, V_{1}, \ldots, V_{j}, \ldots, V_{n}, 0,0,0\right)-H_{i}\left(x, t, V_{1}, \ldots, 0, \ldots, V_{n}, 0,0,0\right) \\
\geq c_{i j}\left(C+\left|\mathbf{u}_{0}\right|_{\infty}\right) .
\end{array}
$$

Furthermore, iterating the process we obtain

$$
\begin{aligned}
& \partial_{t} V_{i}(x, t)+H_{i}\left(x, t, V_{1}, \ldots, V_{j}, \ldots, V_{n}, 0,0,0\right) \\
& \geq\left(C+\left|\mathbf{u}_{0}\right|_{\infty}\right) \sum_{j \in I(n)} c_{i j}+H_{i}(x, t, 0,0,0,0) \\
& \geq C c_{0}-\left|H_{i}(\cdot, \cdot, 0,0,0,0)\right|_{\infty}
\end{aligned}
$$


and taking $C=c_{0}^{-1}|\mathbf{H}(\cdot, \cdot, 0,0,0,0)|_{\infty}$, we get that $\mathbf{V}$ is a supersolution to (1.1)-(1.2) for the problem on $Q$. A lower bound can be obtained similarly by considering a function with the form $U_{i}(x, t)=-C-\left|\mathbf{u}_{0}\right|_{\infty}$ for all $i \in I(n)$. By the comparison principle, the proof is complete.

The comparison principle is used to study steady-state large time behavior for system (1.1). More specifically, we consider functions $\bar{H}_{i}$ and $\bar{K}_{i}$ (with the associated function $\bar{\alpha}_{i}$ ) not depending on the time variable $t$ and satisfying the above conditions in the time-independent formulation for all $i \in I(n)$. Thus, the stationary problem has the form

$$
\bar{H}_{i}\left(x, \mathbf{u}, D u_{i}, D^{2} u_{i}, \overline{\mathcal{T}}_{i}\left(u_{i}\right)\right)=0 \quad \text { in } \mathbb{R}^{d},
$$

where

$$
\overline{\mathcal{T}}_{i}\left(u_{i}, x\right)=\int_{\mathbb{R}^{d}}\left[u_{i}(x+z)-u_{i}(x)-\mathbb{1}_{B}(z)\left\langle D u_{i}(x), z\right\rangle\right] \bar{K}_{i}(x, z) d z .
$$

Following closely the lines in the proofs of Theorem 3.2 and Theorem 4.3, where $c_{0}>0$ in (H2) plays the role of the parabolicity performed by the time derivative, we can conclude the well-posedness of (5.2), given the following result.

Theorem 5.2. Consider the stationary problem (5.2), where for all $i \in$ $I(n), \bar{H}_{i} \in C\left(\mathbb{R}^{d} \times \mathbb{R} \times \mathbb{R}^{d} \times \mathbb{S}^{d} \times \mathbb{R}\right)$, and $\bar{K}_{i} \in C\left(\mathbb{R}^{d} \times\left(\mathbb{R}^{d} \backslash\{0\}\right)\right)$ satisfy the conditions (K)-(S), (H1), (F1), (F3), (F2), or (F2)' and (H2) with $c_{0}>0$, in the time-independent formulation. Then, there exists a unique viscosity solution $\mathbf{u} \in C\left(\mathbb{R}^{d}, \mathbb{R}^{n}\right)$ for (5.2) and the following bound holds:

$$
|\mathbf{u}|_{\infty} \leq c_{0}^{-1}|\overline{\mathbf{H}}(\cdot, 0,0,0,0)|_{\infty} .
$$

The uniqueness for the stationary problem leads to a steady-state large time behavior result for parabolic problems as an application of the half-relaxed limits method introduced by Barles and Perthame [8].

Theorem 5.3. Assume there exist vector functions $\overline{\mathbf{H}}, \overline{\mathbf{K}}$, and the hypotheses of Theorems 5.1 and 5.2 hold, such that

$$
H_{i} \rightarrow \bar{H}_{i}, \quad K_{i} \rightarrow \bar{K}_{i} \quad \text { locally uniform as } t \rightarrow \infty .
$$

Then, the unique bounded viscosity solution $\mathbf{u}$ to (1.1)-(1.2) converges locally uniform in $\mathbb{R}^{d}$ to the unique bounded viscosity solution $\mathbf{u}_{\infty}$ of (5.2) as $t \rightarrow+\infty$.

Moreover, if $\mathbf{H}, \mathbf{K}$ are not dependent on $t$, we have the following rate of convergence:

$$
\left|\mathbf{u}_{\infty}-\mathbf{u}(\cdot, t)\right|_{\infty} \leq e^{-c_{0} t}\left|\mathbf{u}_{\infty}-\mathbf{u}_{0}\right|_{\infty}, \quad \text { for all } t \geq 0 .
$$


Proof: Let $\mathbf{u}$ be the solution to (1.1). For each $i \in I(n), u_{i}$ is a bounded function in $Q$ from which the functions

$$
\bar{u}_{i}(x, t)=\limsup _{y \rightarrow x, \epsilon \rightarrow 0} u_{i}(y, t / \epsilon), \quad \underline{u}_{i}(x, t)=\liminf _{y \rightarrow x, \epsilon \rightarrow 0} u_{i}(y, t / \epsilon)
$$

are well defined for each $(x, t) \in Q$. It is worth noting that

$$
\limsup _{y \rightarrow x, \epsilon \rightarrow 0} u_{i}(y, t / \epsilon)=\lim _{\sigma \rightarrow 0, \epsilon \rightarrow 0} \sup \left\{u_{i}\left(y, t / \epsilon^{\prime}\right): y \in B_{\sigma}(x), 0<\epsilon^{\prime}<\epsilon\right\},
$$

and analogously for liminf.

We claim that for each $t_{0}>0$, the functions $x \mapsto \overline{\mathbf{u}}\left(x, t_{0}\right)$ and $x \mapsto$ $\underline{\mathbf{u}}\left(x, t_{0}\right)$ are respectively viscosity sub and supersolutions to (5.2). We will only deal with subsolutions, as the case of supersolutions is similar.

Let $t_{0}>0$ fixed and $i \in I(n), x_{0} \in \mathbb{R}^{d}, \phi \in C_{b}^{2}\left(\mathbb{R}^{d}\right)$ such that $\bar{u}_{i}\left(\cdot, t_{0}\right)-\phi$ has a global, strict maximum point at $x_{0}$. Hence, taking appropriate sequences in the formulation of $\bar{u}_{i}$, we have the existence of $\delta>0, x_{k} \rightarrow x_{0}$, and $\epsilon_{k} \rightarrow 0$ such that, denoting $t_{k}=t_{0} / \epsilon_{k},\left(x_{k}, t_{k}\right)$ is a maximum point of the function $(x, t) \mapsto u_{i}(x, t)-\phi(x)$ in $B_{\delta}\left(x_{0}\right) \times$ $\left(t_{k}-\delta / \epsilon_{k}, t_{k}+\delta / \epsilon_{k}\right)$, satisfying in addition that $u_{i}\left(x_{k}, t_{k}\right) \rightarrow \bar{u}_{i}\left(x_{0}, t_{0}\right)$ as $k \rightarrow \infty$.

Now, since $\mathbf{u}$ is a subsolution to (1.1) and since $\partial_{t} \phi(x)=0$, we can write

$$
H_{i}\left(x_{k}, t_{k}, \mathbf{u}\left(x_{k}, t_{k}\right), D \phi\left(x_{k}\right), D^{2} \phi\left(x_{k}\right), \mathcal{T}_{i, \delta}\left(u_{i}, \phi, x_{k}, t_{k}\right)\right) \leq 0 .
$$

Before continuing we analyze the nonlocal term; hence by $(\mathrm{K})-(\mathrm{S}),(5.3)$, and the dominated convergence theorem we have

$$
\mathcal{T}_{i}\left[B_{\delta}\right]\left(\phi, x_{k}, t_{k}\right)=o_{k}(1)+\overline{\mathcal{T}}_{i}\left[B_{\delta}\right]\left(\phi, x_{0}\right),
$$

where $\overline{\mathcal{T}}_{i}$ is the operator $(1.3)$ with the kernel $\bar{K}_{i}$. Also, we notice that

$$
\begin{aligned}
\mathcal{T}_{i} & {\left[B_{\delta}^{c}\right]\left(u_{i}\left(\cdot, t_{k}\right), \phi, x_{k}, t_{k}\right)-\overline{\mathcal{T}}_{i}\left[B_{\delta}^{c}\right]\left(\bar{u}_{i}\left(\cdot, t_{0}\right), \phi, x_{0}\right) } \\
\leq & 2|\mathbf{u}|_{\infty} \int_{B_{\delta}^{c}}\left|K_{i}\left(x_{k}, t_{k}, z\right)-\bar{K}_{i}\left(x_{0}, z\right)\right| d z \\
& +\int_{B_{\delta}^{c}}\left|\left(u_{i}\left(x_{k}+z, t_{k}\right)-u_{i}\left(x_{k}, t_{k}\right)\right)-\left(\bar{u}_{i}\left(x_{0}+z, t_{0}\right)-\bar{u}_{i}\left(x_{0}, t_{0}\right)\right)\right| \bar{K}_{i}\left(x_{0}, z\right) d z \\
& +\int_{B \backslash B_{\delta}}\left|\left\langle D \phi\left(x_{k}\right), z\right\rangle K_{i}\left(x_{k}, t_{k}, z\right)-\left\langle D \phi\left(x_{0}\right), z\right\rangle \bar{K}_{i}\left(x_{0}, z\right)\right| d z \\
:= & I_{1}+I_{2}+I_{3} .
\end{aligned}
$$


Similarly to the previous case, by using the dominated convergence theorem, the properties $(\mathrm{K})-(\mathrm{S})$, and the uniform convergence of (5.3), we have $I_{1}, I_{3}=o_{k}(1)$. For the term $I_{2}$ we conclude $I_{2} \leq o_{k}(1)$ by using the definition of $\bar{u}_{i}$ and (K)-(S). Then, summarizing the above estimates,

$$
\mathcal{T}_{i, \delta}\left(u_{i}, \phi, x_{k}, t_{k}\right) \leq o_{k}(1)+\overline{\mathcal{T}}_{i, \delta}\left(\bar{u}_{i}, \phi, x_{0}, t_{0}\right)
$$

Using the property (H1) and (5.4), we have

$$
H_{i}\left(x_{k}, t_{k}, \mathbf{u}\left(x_{k}, t_{k}\right), D \phi\left(x_{k}\right), D^{2} \phi\left(x_{k}\right), o_{k}(1)+\overline{\mathcal{T}}_{i, \delta}\left(\bar{u}_{i}, \phi, x_{0}, t_{0}\right)\right) \leq 0 .
$$

Then, considering the above estimate, the smoothness of $\phi$, the continuity of $H_{i}$, taking $k \rightarrow+\infty$, since then $u_{i}\left(x_{k}, t_{k}\right) \rightarrow \bar{u}_{i}\left(x_{0}, t_{0}\right),(5.3)$, the definition of each $\bar{u}_{j}$, and the monotone property (H2), we conclude that

$$
\bar{H}_{i}\left(x_{0}, \overline{\mathbf{u}}\left(x_{0}, t_{0}\right), D \phi\left(x_{0}\right), D^{2} \phi\left(x_{0}\right), \overline{\mathcal{T}}_{i, \delta}\left(\bar{u}_{i}, \phi, x_{0}, t_{0}\right)\right) \leq 0,
$$

which is the desired viscosity inequality.

To conclude the steady-state large time behavior, we apply the comparison principle for the stationary problem (5.2) and we arrive at $\overline{\mathbf{u}} \leq \underline{\mathbf{u}}$, which leads to the local uniform convergence of $\mathbf{u}$ to the unique solution of the stationary problem.

Now, in order to prove the rate of convergence, we notice that, since $\mathbf{H}, \mathbf{K}$ do not depend on $t$, we have that $\mathbf{H}=\overline{\mathbf{H}}, \mathbf{K}=\overline{\mathbf{K}}$ in (5.3). Hence, we consider the function

$$
U_{i}(x, t)=u_{\infty, i}(x)+e^{-c_{0} t}\left|\mathbf{u}_{\infty}-\mathbf{u}_{0}\right|_{\infty}
$$

for all $i \in I(n)$ and $c_{0}>0$ is the constant in (H2).

We claim that $\mathbf{U}$ is a supersolution for system (1.1)-(1.2). In fact, we clearly have $\mathbf{U}(x, 0) \geq \mathbf{u}_{0}(x)$ and we consider $i \in I(n), \delta>0,\left(x_{0}, t_{0}\right) \in$ $Q_{T}, \phi \in C^{2}\left(\bar{Q}_{T}\right)$ such that $\left(x_{0}, t_{0}\right)$ is a strict minimum point of $U_{i}-\phi$ on $\mathcal{C}_{\delta}\left(x_{0}, t_{0}\right)$ satisfying $U_{i}\left(x_{0}, t_{0}\right)=\phi\left(x_{0}, t_{0}\right)$.

On the other hand, taking $\psi(x)=-e^{-c_{0} t_{0}}\left|\mathbf{u}_{\infty}-\mathbf{u}_{0}\right|_{\infty}+\phi\left(x, t_{0}\right)$, we get that $x_{0}$ is a minimum point for the function $u_{\infty, i}-\psi$ in $B_{\delta}\left(x_{0}\right)$. Since $\mathbf{u}_{\infty}$ is a supersolution of $(5.2)$ and $\mathcal{T}_{i, \delta}\left(u_{\infty, i}, \psi, x_{0}\right)=\mathcal{T}_{i, \delta}\left(U_{i}, \phi\left(\cdot, t_{0}\right), x_{0}\right)$, we see that

$$
H_{i}\left(x_{0}, \mathbf{u}_{\infty}\left(x_{0}\right), D \phi\left(x_{0}, t_{0}\right), D^{2} \phi\left(x_{0}, t_{0}\right), \mathcal{T}_{i, \delta}\left(U_{i}, \phi\left(\cdot, t_{0}\right), x_{0}\right)\right) \geq 0 .
$$

In the following computations, we drop the dependence of $H_{i}$ on $x_{0}$, $D \phi\left(x_{0}, t_{0}\right), D^{2} \phi\left(x_{0}, t_{0}\right)$, and $\left.\mathcal{T}_{i, \delta}\left(U_{i}, \phi\left(\cdot, t_{0}\right), x_{0}\right)\right)$, since these variables do not play any role here. Hence by $(\mathrm{H} 2)$ we get that

$$
\begin{aligned}
& H_{i}\left(U_{1}\left(x_{0}, t_{0}\right), \ldots, U_{j}\left(x_{0}, t_{0}\right), \ldots, U_{n}\left(x_{0}, t_{0}\right)\right) \\
& -H_{i}\left(U_{1}\left(x_{0}, t_{0}\right), \ldots, u_{\infty, j}\left(x_{0}\right), \ldots, U_{n}\left(x_{0}, t_{0}\right)\right) \geq c_{i j} e^{-c_{0} t}\left|\mathbf{u}_{\infty}-\mathbf{u}_{0}\right|_{\infty}
\end{aligned}
$$


for all $j \in I(n)$. Iterating the process for each $j \in I(n)$, we can obtain

$$
\begin{aligned}
\partial_{t} U_{i}\left(x_{0}, t_{0}\right)+H_{i} & \left(\mathbf{U}\left(x_{0}, t_{0}\right)\right) \\
& \geq\left(-c_{0}+\sum_{j \in I(n)} c_{i j}\right) e^{-c_{0} t_{0}}\left|\mathbf{u}_{\infty}-\mathbf{u}_{0}\right|_{\infty}+H_{i}\left(\mathbf{u}_{\infty}\left(x_{0}\right)\right) \\
& \geq H_{i}\left(\mathbf{u}_{\infty}\left(x_{0}\right)\right) .
\end{aligned}
$$

By the above computations, we conclude that $\mathbf{U}$ is a supersolution to $(1.1)$.

In the same way, the function

$$
V_{i}(x, t)=u_{\infty, i}(x)-e^{-c_{0} t}\left|\mathbf{u}_{\infty}-\mathbf{u}_{0}\right|_{\infty}, \quad \forall i \in I(n),
$$

is a subsolution to (1.1), and the result follows by the comparison principle.

Acknowledgments. The author was supported by Escuela Politécnica Nacional, Proyecto PII-DM-2019-01. The author thanks the anonymous referees for their comments, which have resulted in a new version that gives more value to our results.

\section{References}

[1] O. Alvarez and A. Tourin, Viscosity solutions of nonlinear integro-differential equations, Ann. Inst. H. Poincaré Anal. Non Linéaire 13(3) (1996), 293-317. DOI : $10.1016 / \mathrm{S} 0294-1449$ (16) 30106-8.

[2] M. Arisawa, Homogenization of a class of integro-differential equations with Lévy operators, Comm. Partial Differential Equations 34(7) (2009), 617-624. DOI : $10.1080 / 03605300902963518$.

[3] J. BAE, Regularity for fully nonlinear equations driven by spatial-inhomogeneous nonlocal operators, Potential Anal. 43(4) (2015), 611-624. DOI:10.1007/ s11118-015-9488-z.

[4] J. Bae and M. Kassmann, Schauder estimates in generalized Hölder spaces, Preprint (2015). arXiv:1505.05498.

[5] G. Barles, R. Buckdahn, and E. Pardoux, Backward stochastic differential equations and integral-partial differential equations, Stochastics Stochastics Rep. 60(1-2) (1997), 57-83. DOI : 10.1080/17442509708834099.

[6] G. Barles and C. Imbert, Second-order elliptic integro-differential equations: viscosity solutions' theory revisited, Ann. Inst. H. Poincaré Anal. Non Linéaire 25(3) (2008), 567-585. DOI : 10.1016/j. anihpc.2007.02.007.

[7] G. Barles, S. Koike, O. Ley, and E. Topp, Regularity results and large time behavior for integro-differential equations with coercive Hamiltonians, Calc. Var. Partial Differential Equations 54(1) (2015), 539-572. DOI: 10.1007/ s00526-014-0794-x.

[8] G. Barles and B. Perthame, Exit time problems in optimal control and vanishing viscosity method, SIAM J. Control Optim. 26(5) (1988), 1133-1148. DOI : $10.1137 / 0326063$. 
[9] R. F. BAss, Uniqueness in law for pure jump Markov processes, Probab. Theory Related Fields 79(2) (1988), 271-287. DOI : 10.1007/BF00320922.

[10] R. F. BAss, Occupation time densities for stable-like processes and other pure jump Markov processes, Stochastic Process. Appl. 29(1) (1988), 65-83. DOI : 10. 1016/0304-4149 (88) 90028-2.

[11] R. F. BAss, Regularity results for stable-like operators, J. Funct. Anal. 257(8) (2009), 2693-2722. DOI : 10.1016/j.jfa.2009.05.012.

[12] R. F. BASS AND M. KASSMAnN, Hölder continuity of harmonic functions with respect to operators of variable order, Comm. Partial Differential Equations 30(8) (2005), 1249-1259. DOI : 10.1080/03605300500257677.

[13] R. F. BASS AND M. KASSMAnN, Harnack inequalities for non-local operators of variable order, Trans. Amer. Math. Soc. 357(2) (2005), 837-850. DOI: 10.1090/ S0002-9947-04-03549-4.

[14] R. F. BAss And D. A. Levin, Harnack inequalities for jump processes, Potential Anal. 17(4) (2002), 375-388. DOI : 10.1023/A:1016378210944.

[15] F. E. Benth, K. H. Karlsen, and K. Reikvam, Optimal portfolio selection with consumption and nonlinear integro-differential equations with gradient constraint: a viscosity solution approach, Finance Stoch. 5(3) (2001), 275-303. DOI : $10.1007 /$ PL00013538.

[16] J. Bertoin, "Lévy Processes", Cambridge Tracts in Mathematics 121, Cambridge University Press, Cambridge, 1996.

[17] L. Caffarelli and L. Silvestre, Regularity theory for fully nonlinear integrodifferential equations, Comm. Pure Appl. Math. 62(5) (2009), 597-638. DOI : 10. 1002/cpa. 20274.

[18] F. Camilli, O. Ley, and P. Loreti, Homogenization of monotone systems of Hamilton-Jacobi equations, ESAIM Control Optim. Calc. Var. 16(1) (2010), 58-76. DOI : $10.1051 / \mathrm{cocv}: 2008061$.

[19] P. Cardaliaguet, Solutions de viscosité d'équations elliptiques et paraboliques non linéaires, Notes d'un cours de DEA, Université de Rennes (2004).

[20] X. Chen, Z.-Q. Chen, And J. WAng, Heat kernel for non-local operators with variable order, Stochastic Process. Appl. 130(6) (2020), 3574-3647. DOI: 10. 1016/j.spa.2019.10.004.

[21] Z.-Q. Chen And J.-M. Wang, Perturbation by non-local operators, Ann. Inst. Henri Poincaré Probab. Stat. 54(2) (2018), 606-639. DOI : 10.1214/16-AIHP816.

[22] M. G. Crandall and P.-L. Lions, Viscosity solutions of Hamilton-Jacobi equations, Trans. Amer. Math. Soc. 277(1) (1983), 1-42. DOI: 10.2307/1999343.

[23] H. Engler and S. M. Lenhart, Viscosity solutions for weakly coupled systems of Hamilton-Jacobi equations, Proc. London Math. Soc. (3) 63(1) (1991), 212-240. DOI : 10.1112/plms/s3-63.1.212.

[24] H. IsHiI, Perron's method for Hamilton-Jacobi equations, Duke Math. J. 55(2) (1987), 369-384. DOI : 10.1215/S0012-7094-87-05521-9.

[25] H. Ishit AND S. KoIKe, Viscosity solutions for monotone systems of secondorder elliptic PDEs, Comm. Partial Differential Equations 16(6-7) (1991), 1095-1128. DOI : 10.1080/03605309108820791.

[26] N. JACOB, Further pseudodifferential operators generating Feller semigroups and Dirichlet forms, Rev. Mat. Iberoamericana 9(2) (1993), 373-407. DOI : 10.4171/ RMI/141.

[27] N. JACOB, A class of Feller semigroups generated by pseudo-differential operators, Math. Z. 215(1) (1994), 151-166. DOI : 10.1007/BF02571704. 
[28] N. JaCOB AND H.-G. LeOPold, Pseudo-differential operators with variable order of differentiation generating Feller semigroups, Integral Equations Operator Theory 17(4) (1993), 544-553. DOI : 10.1007/BF01200393.

[29] E. R. Jakobsen and K. H. Karlsen, Continuous dependence estimates for viscosity solutions of integro-PDEs, J. Differential Equations 212(2) (2005), 278-318. DOI : $10.1016 / \mathrm{j}$.jde. 2004.06.021.

[30] E. R. JaKobsen and K. H. KARLSEn, A "maximum principle for semicontinuous functions" applicable to integro-partial differential equations, NoDEA Nonlinear Differential Equations Appl. 13(2) (2006), 137-165. DOI: 10.1007/ s00030-005-0031-6.

[31] K. Kikuchi And A. Negoro, On Markov process generated by pseudodifferential operator of variable order, Osaka J. Math. 34(2) (1997), 319-335. DOI: 10. 18910/12776.

[32] Y.-C. KIm AND K.-A. LeE, Regularity results for fully nonlinear integrodifferential operators with nonsymmetric positive kernels, Manuscripta Math. 139(3-4) (2012), 291-319. DOI : 10.1007/s00229-011-0516-z.

[33] Y.-C. KIm AND K.-A. LeE, Regularity results for fully nonlinear integrodifferential operators with nonsymmetric positive kernels: subcritical case, $P o-$ tential Anal. 38(2) (2013), 433-455. DOI : 10.1007/s11118-012-9280-2.

[34] S. Kolke, Uniqueness of viscosity solutions for monotone systems of fully nonlinear PDEs under Dirichlet condition, Nonlinear Anal. 22(4) (1994), 519-532. DOI : $10.1016 / 0362-546 \mathrm{X}(94) 90172-4$.

[35] H. G. Leopold of Jena, On a class of function spaces and related pseudodifferential operators, Math. Nachr. 127(1) (1986), 65-82. DOI : 10.1002/mana. 19861270106.

[36] H.-G. Leopold, On Besov spaces of variable order of differentiation, Z. Anal. Anwendungen 8(1) (1989), 69-82. DOI : 10.4171/ZAA/337.

[37] H.-G. LEOPOLD, On function spaces of variable order of differentiation, Forum Math. 3(1) (1991), 1-21. DOI : 10.1515/form.1991.3.1.

[38] O. Ley and V. D. NGuYen, Gradient bounds for nonlinear degenerate parabolic equations and application to large time behavior of systems, Nonlinear Anal. 130 (2016), 76-101. DOI : 10.1016/j.na.2015.09.012.

[39] D. LuO AND J. WANG, Coupling by reflection and Hölder regularity for non-local operators of variable order, Trans. Amer. Math. Soc. 371(1) (2019), 431-459. DOI : $10.1090 / \operatorname{tran} / 7259$.

[40] H. Mitake and H. V. Tran, A dynamical approach to the large-time behavior of solutions to weakly coupled systems of Hamilton-Jacobi equations, J. Math. Pures Appl. (9) 101(1) (2014), 76-93. DOI : 10.1016/j.matpur.2013.05.004.

[41] A. Negoro, Stable-like processes: construction of the transition density and the behavior of sample paths near $t=0$, Osaka J. Math. 31(1) (1994), 189-214. DOI : $10.18910 / 11079$.

[42] B. ØKsendal And A. Sulem, "Applied Stochastic Control of Jump Diffusions", Universitext, Springer-Verlag, Berlin, 2005. DOI : 10.1007/b137590.

[43] H. PнAм, Optimal stopping of controlled jump diffusion processes: a viscosity solution approach, J. Math. Systems Estim. Control 8(1) (1998), 27 pp.

[44] K.-I. Sato, "Lévy Processes and Infinitely Divisible Distributions", Translated from the 1990 Japanese original, Revised by the author, Cambridge Studies in Advanced Mathematics 68, Cambridge University Press, Cambridge, 1999. 
[45] A. SaYAh, Equations d'Hamilton-Jacobi du premier ordre avec termes intégrodifférentiels. Partie I: Unicité des solutions de viscosité, Comm. Partial Differential Equations 16(6-7) (1991), 1057-1074. DOI : 10.1080/03605309108820789. Partie II: Existence de solutions de viscosité, Comm. Partial Differential Equations 16(6-7) (1991), 1075-1093. DOI : 10.1080/03605309108820790.

[46] L. Silvestre, Hölder estimates for solutions of integro-differential equations like the fractional Laplace, Indiana Univ. Math. J. 55(3) (2006), 1155-1174. DOI : 10.1512/iumj .2006.55.2706.

[47] H. M. Soner, Optimal control with state-space constraint. II, SIAM J. Control Optim. 24(6) (1986), 1110-1122. DOI : 10.1137/0324067.

[48] E. Topp AND M. YANGARI, Existence and uniqueness for parabolic problems with Caputo time derivative, J. Differential Equations 262(12) (2017), 6018-6046. DOI : $10.1016 / j \cdot j$ de. 2017.02 .024 .

[49] M. Tsuchiya, Lévy measure with generalized polar decomposition and the associated SDE with jumps, Stochastics Stochastics Rep. 38(2) (1992), 95-117. DOI : $10.1080 / 17442509208833748$.

[50] J.-M. WAng, Laplacian perturbed by non-local operators, Math. Z. 279(1-2) (2015), 521-556. DOI : $10.1007 / \mathrm{s} 00209-014-1380-9$.

[51] L. WANG AND B. ZHANG, Infinitely many solutions for Kirchhoff-type variableorder fractional Laplacian problems involving variable exponents, Appl. Anal. (2019), pp. 1-18. DOI : 10.1080/00036811.2019.1688790.

[52] W. A. Woyczyński, Lévy processes in the physical sciences, in: "Lévy Processes", Birkhäuser Boston, Boston, MA, 2001, pp. 241-266.

[53] M. Xiang, B. Zhang, and D. Yang, Multiplicity results for variable-order fractional Laplacian equations with variable growth, Nonlinear Anal. 178 (2019), 190-204. DOI : 10.1016/j.na.2018.07.016.

Departamento de Matemática, Escuela Politécnica Nacional, Ladrón de Guevara E11253, P.O. Box 17-01-2759, Quito, Ecuador

E-mail address: miguel.yangari@epn.edu.ec

Received on February 26, 2020.

Accepted on December 3, 2020. 